\title{
Kernos
}

Revue internationale et pluridisciplinaire de religion grecque antique

$24 \mid 2011$

Varia

\section{Quelles offrandes faisait-on à Artémis dans son sanctuaire de Thasos?}

Jean-Jacques Maffre et Anne Tichit

\section{(apenEdition \\ Journals}

Édition électronique

URL : http://journals.openedition.org/kernos/1961

DOI : 10.4000/kernos. 1961

ISSN : 2034-7871

Éditeur

Centre international d'étude de la religion grecque antique

Édition imprimée

Date de publication : 1 janvier 2011

Pagination : 137-164

ISSN : 0776-3824

Référence électronique

Jean-Jacques Maffre et Anne Tichit, «Quelles offrandes faisait-on à Artémis dans son sanctuaire de Thasos? », Kernos [En ligne], 24 | 2011, mis en ligne le 01 février 2014, consulté le 04 mai 2019. URL http://journals.openedition.org/kernos/1961; DOI : 10.4000/kernos.1961 


\title{
Quelles offrandes faisait-on à Artémis dans son sanctuaire de Thasos?
}

\author{
À la mémoire de Nicole Weill.
}

\begin{abstract}
Résumé : Les travaux et publications récents concernant le très abondant matériel de l'Artémision de Thasos permettent de proposer une première vue d'ensemble. Nous sommes ici dans une situation privilégiée puisque nous savons par les sources écrites qu'il s'agit d'un sanctuaire consacré à Artémis. Le matériel votif du sanctuaire permet-il alors de dégager un aspect particulier de la personnalité d'Artémis à Thasos ? Si quelques offrandes sont clairement le signe d'une dévotion de jeunes filles et de femmes ou sont directement à mettre en relation avec les attributions d'Artémis, force est de constater, à ce point d'avancée des recherches, l'absence d'offrandes vraiment originales, et en particulier le nombre très réduit de représentations de la déesse dans son sanctuaire, ce qui ne permet guère de déterminer une fonction précise de celle-ci à Thasos.
\end{abstract}

Abstract: Recent research and publications on the very numerous votive offerings found in the Thasian Artemision allow us to provide a preliminary overview. The context is favourable, since written evidence informs us that this sanctuary was dedicated to Artemis. Do the offerings found in the sanctuary allow us to point out a particular aspect of the Artemis' personality in Thasos? Some offerings clearly indicate that girls and women worshipped the goddess, or else they can be directly related to Artemis' prerogatives. However, we can infer from current investigation that no truly original offerings are attested and that the goddess herself is very rarely represented on offerings in her shrine. Thus this evidence does not allow us to define a precise function of Artemis in Thasos.

Il n'est pas toujours facile de déterminer avec précision, en l'absence de données écrites, à quelle divinité était consacré un lieu de culte antique, et l'on constate que l'identification de certains sanctuaires mis au jour lors de fouilles archéologiques a donné lieu, même dans un passé récent, à des hésitations qui n’ont pu être levées que grâce à des découvertes épigraphiques ou à des secours apportés par des sources littéraires. Un premier exemple est fourni par l'Aphrodision d'Argos, dont seuls un passage de Pausanias, désormais mieux compris, et quelques graffiti inscrits sur des fragments de vases votifs ont permis l'identification lors du dégagement, par l'École française d'Athènes, entre 1967 et 1974, d'un petit téménos proche de l'odéon et riche de nombreux 
Fig. 1.

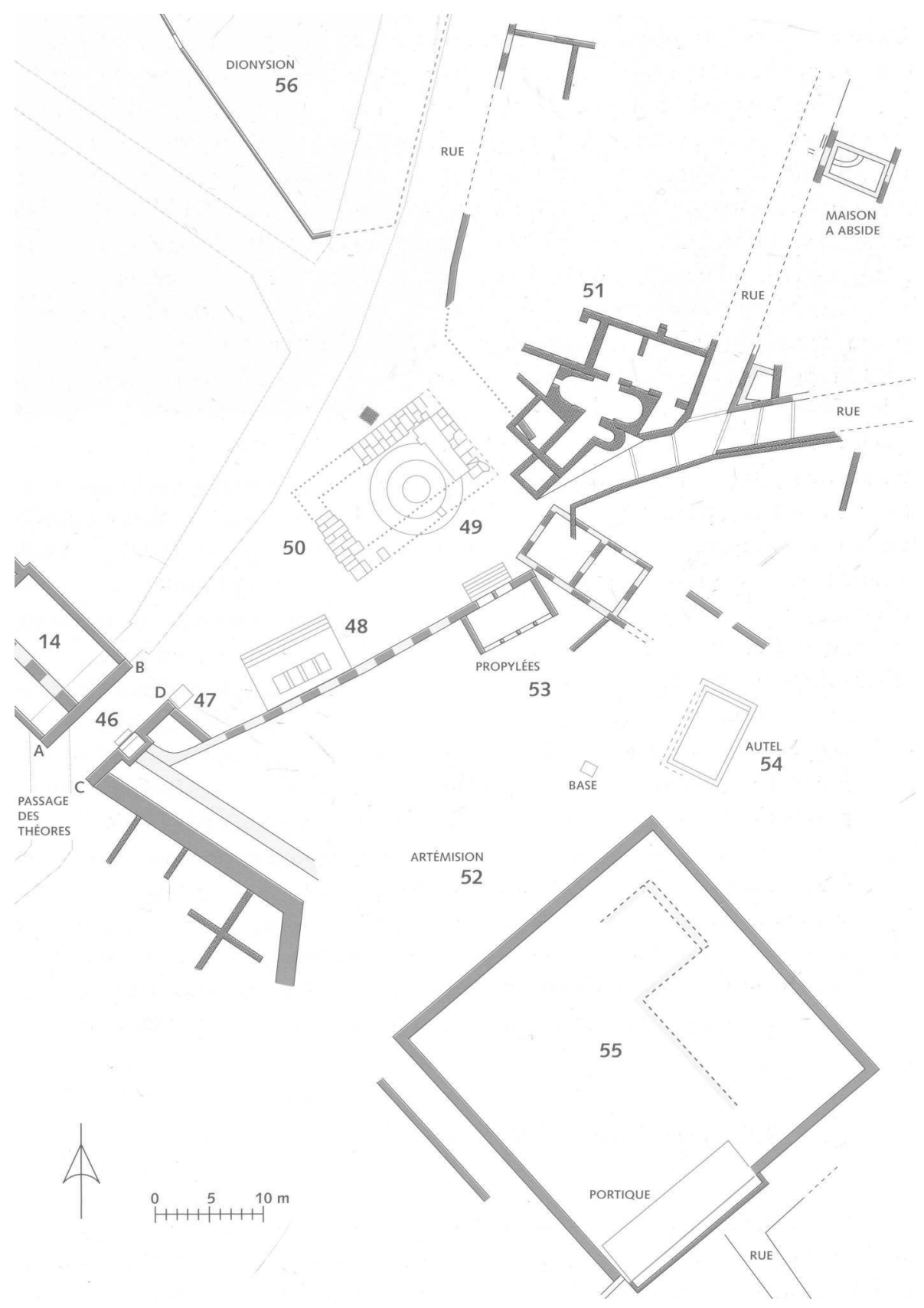


ex-voto ${ }^{1}$. Autre exemple : un sanctuaire mis au jour en 1970-71 à ÉpidamneDyrrhachion, d'abord attribué par les fouilleurs albanais, au vu des nombreuses figurines de terre cuite exhumées, à Aphrodite, puis peut-être à Déméter et Korè, a été définitivement identifié comme consacré à Artémis - ce qu'avait déjà supposé l'équipe franco-albanaise en charge de l'étude du matériel lorsqu'on a repéré au nettoyage, sur certains fragments de vases en argile, des dédicaces, peintes ou incisées, en l'honneur de cette déesse ${ }^{2}$.

À Thasos, c'est depuis la découverte, au début du $\mathrm{XX}^{\mathrm{e}}$ siècle, de bases de statues féminines porteuses d'une dédicace à Artémis, puis, dans le même secteur, de trois inscriptions lapidaires qui sont elles aussi des dédicaces à cette déesse, que la localisation de l'Artémision est assurée, au pied des premières pentes de l'Acropole, à proximité de l'Agora (Fig. 1)3. Dans le matériel votif luimême, deux fragments porteurs de graffiti confirment l'identification du sanctuaire: ce sont des dédicaces à Artémis, brèves et incomplètes mais assurées. Pour l'une d'entre elles, A. Jacquemin proposait à juste titre la lecture APTE $[\mathrm{MI} \Delta \mathrm{I}]$; on peut suggérer la même restitution pour l'autre (Fig. 2)4.

Des fouilles d'une grande ampleur ont été menées sur la terrasse supérieure du sanctuaire dans les années 1957-1961 par la regrettée N. Weill et par Fr. Salviat, puis sur la partie inférieure, en 1965, par Fr. Croissant. Entre 1975 et 1985, Fr. Salviat et J.-J. Maffre, A. Jacquemin et J. Perrault, ont fouillé à la fois

1 Voir en dernier lieu, avec un excellent résumé des données relatives à ce sanctuaire, Fr. Croissant, «Identification d'une déesse : questions sur l'Aphrodite argienne », in Cl. PRÊTRE, (éd.), avec la collaboration de St. HuYSECOM-HAXHI, Le donateur, l'offrande et la déesse. Systèmes votifs dans les sanctuaires de déesses du monde grec (Actes du 31e colloque international organisé par l'UMR HALMAIPEL, Lille 3, 13-15/12/2007), Liège, 2009 (Kernos, Suppl. 23), p. 181-202.

2 Voir les indications données et les mises au point proposées récemment par A. MuLLER, F. TARTARI, «L'Artémision de Dyrrhachion : identification, offrandes, topographie », CRAI 2006/1, p. 78-79; M. Muller-Dufeu, E. SheHI, "Skyphoi avec dédicaces peintes de l'Artémision d’Épidamne-Dyrrhachion », BCH 133 (2009) [2011], p. 99-112.

3 Voir Th. MACRIDY, "Un hiéron d'Artémis Pôlô à Thasos», JdI 27 (1912), p. 1-19; A.J. ReInACH, CRAI 1912, p. 222-235. Les dédicaces à Artémis Pôlô sur des bases de statues sont publiées dans IG, XII Suppl. (1939), 382 et 383. Statues et bases sont conservées au Musée archéologique d'Istanbul: voir G. MENDEL, Musées impériaux ottomans. Catalogue des sculptures grecques, romaines et byzantines I, Constantinople, 1912, p. 336-348, nos 130-137; l'auteur précise (p. 336) : les statues « étaient placées sur une série de bases contiguës, dans une sorte de portique dont la façade se développe d'est en ouest, ouverte du côté nord et adossée au rocher du côté sud; les inscriptions gravées sur plusieurs des bases montrent que ce portique faisait partie d'un sanctuaire d'Artémis Pôlô ». - Une autre dédicace à Artémis (sans épiclèse) trouvée dans le même secteur a été publiée par J. Pouilloux, Recherches sur l'histoire et les cultes de Thasos, Paris, 1954 ('tThas III), p. 329, n 124bis. Deux autres inscriptions lapidaires mises au jour en 1957 et 1960 toujours dans le même secteur, l'une du IVe s. av. J.-C., l'autre du début du Ier s. ap. J.-C., sont aussi des dédicaces à Artémis (sans épiclèse) : P. BERnard, Fr. SAlviat, "Inscriptions de Thasos », BCH 86 (1962), p. 592, nos 13-14.

${ }^{4}$ Le premier graffito est signalé et illustré par A. JACQUEMIN, «Chronique des fouilles », $B C H$ 106 (1982), p. 665 et 667, fig. 40; l'autre, inédit, se trouve sur un fragment de lèvre d'une coupe attique à vernis noir, sans doute de la première moitié du ve siècle, trouvé en 1979 près de l'autel mis au jour sur la terrasse inférieure du sanctuaire (Fig. 2). 
sur la terrasse supérieure et en contrebas, dans un terrain où les limites du téménos ont été partiellement atteintes, du côté Nord. Le sanctuaire s'est en effet développé sur deux terrasses. Celle du bas fut aménagée dans la 2e moitié du VI ${ }^{\mathrm{e}}$ siècle av. J.-C. Son mur de soutènement fut en partie arasé au IVe siècle av. J.-C. pour être remplacé par un mur en grand appareil, en même temps qu'étaient mis en place des propylées à portes jumelles. Au Sud-Est de l'entrée se dressait le grand autel, sans doute construit au début du Ve siècle, d'après les données du remblai qui servit à son aménagement et qui contenait une grande quantité d'ossements et d'ex-voto brisés d'époque archaïque. La terrasse supérieure était entourée, dans son état hellénistique, d'un mur de péribole formant un carré de 100 pieds de côté. Le téménos ainsi délimité recouvrait un mur d'époque archaïque, sans doute du début du VI ${ }^{\mathrm{e}}$ siècle, dont les vestiges constituent le témoignage architectural le plus ancien du secteur.

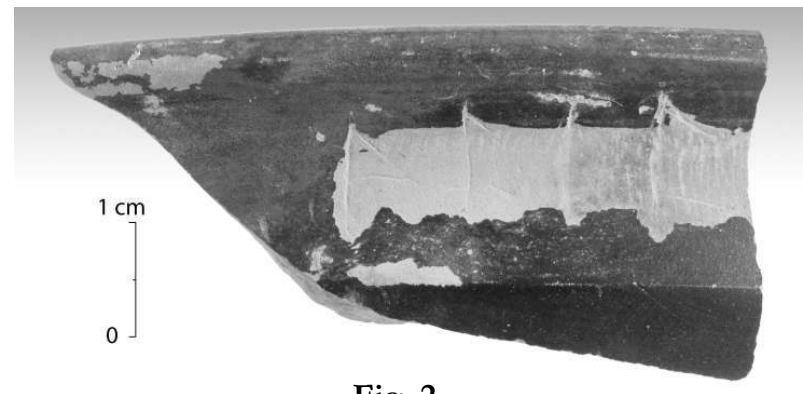

Fig. 2

Le sanctuaire fut fréquenté tout au long de l'Antiquité, comme l'attestent plusieurs témoignages écrits, épigraphiques ou littéraires ${ }^{5}$, complétés par les données archéologiques. Il fut manifestement installé peu après l'arrivée des colons, sans doute sur la terrasse supérieure, d'où proviennent les plats orientalisants du VII siècle, qui sont les objets les plus anciens à avoir été découverts et qui constituent les premiers ex-voto.

Le matériel mis au jour se laisse dater entre la haute époque archaïque et l'époque romaine impériale, avec une nette majorité d'objets du VI ${ }^{\mathrm{e}} \mathrm{s}$. av. J.-C., époque à laquelle le sanctuaire a connu assurément une grande fréquentation. Les offrandes, très nombreuses, appartiennent à des catégories variées : vases, figurines de terre cuite, petits objets en métal, en verre, en os, en ivoire ou en d'autres matériaux plus rares ${ }^{6}$. Ce matériel est si abondant et si riche (quoique le

5 L'Artémision est attesté littérairement au moins dès l'époque classique. Comme le rappelle Fr. Salviat en publiant des décrets pour Epié, fille de Dionysios, datables de la fin de l'époque hellénistique, «les mentions de l'Artémision dans ce texte en sont les premières attestations épigraphiques; elles doivent être rapprochées d'un unique témoignage littéraire, un passage du recueil des 'pidémies d'Hippocrate (III, 102), où le domicile d'un malade est repéré par rapport au sanctuaire » (BCH 83, 1959, p. 376).

${ }^{6}$ Sur les fouilles de l'Artémision et les trouvailles qu'elles ont entraînées, voir notamment les Chroniques publiées dans le BCH: BCH 82 (1958), p. 808-815, BCH 83 (1959), p. 775-781, BCH 84 (1960), p. 856-862, BCH 85 (1961), p. 919-930, BCH 90 (1966), p. 944-959, BCH 100 (1976), p. 774-784, BCH 101 (1977), p. 686-692, BCH 102 (1978), p. 821-829, BCH 104 (1980), p. 726- 
plus souvent très fragmentaire) qu'il n'a pas encore été possible d'en faire connaître plus qu'une faible partie, même si la préparation d'une publication, par catégories de matériel, est maintenant bien avancée.

La présentation de pièces particulièrement remarquables a été faite par N. Weill et Fr. Salviat, puis par J.-J. Maffre, dans des articles du BCH ou dans des Actes de colloques ${ }^{7}$. La publication partielle de quelques séries de matériel trouvé surtout lors des fouilles anciennes a déjà été effectuée ${ }^{8}$. L'étude pour publication

730, BCH 105 (1981), p. 932-941, BCH 110 (1986), p. 790-801. Pour une première synthèse sur les fouilles anciennes, voir Fr. SALviat, N. Weill, "The Sanctuary of Artemis in Thasos", Archaeology 13 (1960), p. 97-104. Voir aussi plus récemment Y. GRANDJEAN, Fr. SALVIAT et al., Guide de Thasos, Paris, 2000, p. 89-91, p. 272-282 (sculptures et figurines en terre cuite), p. 283-296 (céramique).

${ }^{7}$ N. WEILL, «Céramique thasienne à figures noires », BCH 83 (1959), p. 430-454, pl. 24-26; Fr. Salviat, N. Weill, «Plat aux lions affrontés de l'Artémision thasien : art 'rhodien' et art cycladique au VII e siècle ", BCH 85 (1961), p. 98-122, pl. 3-4; EID., "Un plat du VII siècle à Thasos: Bellérophon et la Chimère », BCH 84 (1960), p. 347-386, pl. 4-6; Fr. SALVIAT, "La colonisation grecque dans le Nord de l'Égée. Céramique parienne orientalisante. Céramiques précoloniales à Thasos ", in Le rayonnement des civilisations grecque et romaine sur les cultures périphériques (Actes du $8^{e}$ Congrès international d'Archéologie classique, Paris, 1963), Paris, 1965, p. 299-303, pl. 55-58; ID., "La céramique de style chiote à Thasos », in Les céramiques de la Grèce de l'Est et leur diffusion en Occident (Actes du colloque tenu au Centre J. Bérard, Institut français de Naples, 6-9/7/1976), Paris/ Naples, 1978, p. 87-92, pl. 45-52; ID., «La céramique thasienne orientalisante et l'origine des vases 'méliens'», in Les Cyclades. Matériaux pour une étude de géographie historique (Actes de la Table ronde réunie à l'Univ. de Dijon, 11-13/3/1982), Paris, 1983, p. 185-190; ID., "Plats creux insulaires à décor orientalisant à Thasos", ibid., p. 201-216. - J.-J. MAFFre, "Céramique attique à décor mythologique trouvée à l'Artémision de Thasos ", BCH Suppl. V: Thasiaca, 1979, p. 11-74; ID., "Chachrylion, Euphronios et quelques-uns de leurs contemporains à Thasos», in J. ChristianSen, T. MeLander (éds), Proceedings of the 3rd Symposium on Ancient Greek and Related Pottery, Copenhagen, 1987, Copenhague, 1988, p. 379-389; ID., «Les importations de céramique attique à

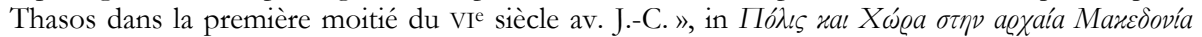
(Actes du colloque à la mémoire de D. Lazaridis, Kavala, 9-11/5/1986), Thessalonique, 1990, p. 245265; ID., "Une nouvelle représentation de potiers au travail : un skyphos attique à figures noires de l'Artémision de Thasos ", in Thasos: Matières premières et technologie, de la prébistoire à nos jours (Actes du Colloque International, Liménaria, 26-29/9/1995), Athènes, 1999, p. 269-278; ID., "Coupes attiques à figures rouges trouvées à Thasos », in J.H. OAKLEY, O. PALAGIA (éds), Athenian Potters and Painters II, Oxford, 2009, p. 181-191 + pl. couleur 11.

8 Pour une partie des terres cuites archaïques, voir N. WeILL, La plastique archaïque de Thasos. Figurines et statues de terre cuite de l'Artémision, I : le haut archaïme, Athènes/Paris, 1985 ('tThas, XI). Un certain nombre de protomés féminines archaïques trouvées entre 1958 et 1965 a été pris en compte dans l'ouvrage général consacré à cette catégorie d'objets par Fr. CROISSANT, Les protomés féminines archä̈ques. Recherches sur les représentations du visage dans la plastique grecque de 550 à 480 av. J.-C., Athènes/Paris, 1983 (BEFAR, 250) : l'index renvoie à 50 protomés de Thasos, pour la plupart de l'Artémision. - Un relief provenant probablement de l'Artémision est présenté par B. HoLTZMANN, La sculpture de Thasos. Corpus des reliefs I : Reliefs à thème divin, Athènes/Paris, 1994 ('tThas, XV), p. 127131, pl. 43a, n 57 . - La céramique thasienne à figures noires, dont bien des pièces ont été trouvées à l'Artémision, a été étudiée par A. Coulié, La céramique thasienne à figures noires, Athènes, 2002 ('tThas XIX). - L'ensemble des figurines archaïques vient d'être publié : St. HUYSECOM-HAXHI, Les figurines en terre cuite de l'Artémision de Thasos: artisanat et piété populaire à l'époque de l'Archaïsme mûr et récent, Athènes, 2009 (' tThas, XXI). 
d'autres catégories a été confiée à divers chercheurs ${ }^{9}$. Nous venons de donner un bref compte rendu des recherches menées depuis quelques décennies sur la céramique attique ${ }^{10}$. Nous n'avons en outre jamais refusé à des chercheurs lancés dans des études d'ensemble de tel ou tel peintre de vases ou de telle ou telle catégorie d'objets l'accès au matériel, même inédit, trouvé dans les fouilles françaises de Thasos, principalement de l'Artémision ${ }^{11}$.

Comme, malgré tout, une grande partie du matériel de l'Artémision thasien reste peu connue, il nous a paru intéressant de proposer une sorte d'état de la question en 2010/2011, avec des rappels et des éléments nouveaux d'information accompagnés de quelques réflexions générales, pour tenter d'offrir de ce matériel votif une vue d'ensemble qui n'a jamais encore été donnée. Puisque nous savons, grâce à des témoignages sûrs, qu'il s'agit d'un sanctuaire d'Artémis et que nous sommes donc a priori dans une situation privilégiée d'étude du matériel votif, pouvons-nous déterminer une spécificité des offrandes? Pouvons-nous dégager à partir de celles-ci quelques aspects de la personnalité de la déesse à Thasos ou entrevoir ses fonctions dans son sanctuaire? Pouvons-nous souscrire à l'opinion de N. Weill qui écrivait naguère : «Le matériel votif considéré dans son ensemble sur un site déterminé permet d'observer comment les fidèles ont imaginé une personnalité divine, et quelles images ils ont choisi de lui consacrer $»^{12}$ ?

${ }^{9}$ M.-Fr. Billot étudie les terres cuites architecturales. Cl. Prêtre prépare la publication du petit matériel votif, J.-J. Maffre celle d'une grande partie de la céramique attique, J. Perrault celle des coupes attiques archaïques à palmettes. Parmi les jeunes membres de l'École française, A. Tichit s'est vu confier la publication des coupes attiques du type de Cassel. Une des collaboratrices les plus anciennes au travail d'étude de la céramique attique de Thasos, Chr. Walter, publiera les coupes de Droop. Notre collègue hollandais K. Neeft se charge de la céramique corinthienne. Une équipe du laboratoire Halma-Ipel - UMR 8164 (Lille 3, CNRS, MCC), sous la direction d'A. Muller, s'occupe de l'ensemble des figurines de terre cuite.

${ }^{10} \mathrm{~J}$.-J. MAFFre, A. TiChit, Chr. WALTER, «Trente ans de recherches sur la céramique attique

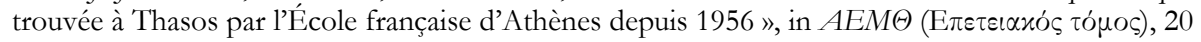
Xeóvıx, Thessalonique, 2009, p. 185-203.

${ }^{11} \mathrm{H}$. Brijder a vu la plupart des fragments de coupes des Cômastes et de coupes de Siana trouvés à Thasos et les a intégrés dans ses belles publications sur ces deux catégories de vases : H.A.G. BrijDer, Siana Cups I and Komast Cups, Amsterdam, 1983 (Allard Pierson Series, 4); ID., Siana Cups II : The Heidelberg Painter, Amsterdam, 1991 (Allard Pierson Series, 8); ID., Siana Cups III : The Red-black Painter, Griffin-bird Painter and Siana Cups resembling Lip-cups, Amsterdam, 2000 (Allard Pierson Series, 13). S. Fritzilas a pu prendre en compte les fragments de skyphoi attribuables au

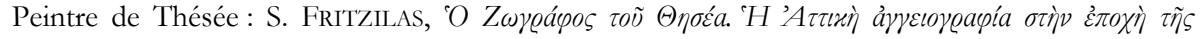

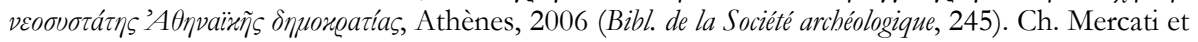
P. Badinou ont été autorisées à mentionner et à illustrer les fragments d'épinétra: P. BADINOU, La laine et le parfum. 'pinetra et alabastres, forme, iconographie et fonction: recherche de céramique attique féminine, Leuven, 2003; Ch. MerCATI, Epinetron, Storia di una forma ceramica fra archeologia e cultura, Città di Castello, 2003.

12 N. WeILL, «Images d'Artémis à l'Artémision de Thasos", in EI $\triangle \Omega \Lambda O \Pi O I L A$. Actes du Colloque sur les problèmes de l'image dans le monde méditerranéen classique, Lourmarin, 1982, Rome, 1985, p. 137. 


\section{Tout le matériel découvert à l'Artémision de Thasos est-il votif?}

On a souvent tendance, d'ordinaire implicitement, à considérer sans discussion comme votif tout objet trouvé dans un sanctuaire. Dans le cas de Thasos, peut-on estimer que tous les objets découverts à l'Artémision sont des offrandes faites à la déesse ${ }^{13}$, ou bien n'y a-t-il qu'une partie du matériel qui soit votif ? En effet, la plupart des objets n'ont pas été trouvés en place, mais dans des remblais, souvent eux-mêmes perturbés par les aménagements du terrain à diverses époques. Il reste cependant probable qu'une grande partie du matériel mis au jour était votif. L'abondance des figurines, des protomés et aussi des grandes statues en terre cuite, la plupart de type féminin, témoigne du souci d'honorer une divinité féminine dont nous avons vu qu'elle avait nom Artémis.

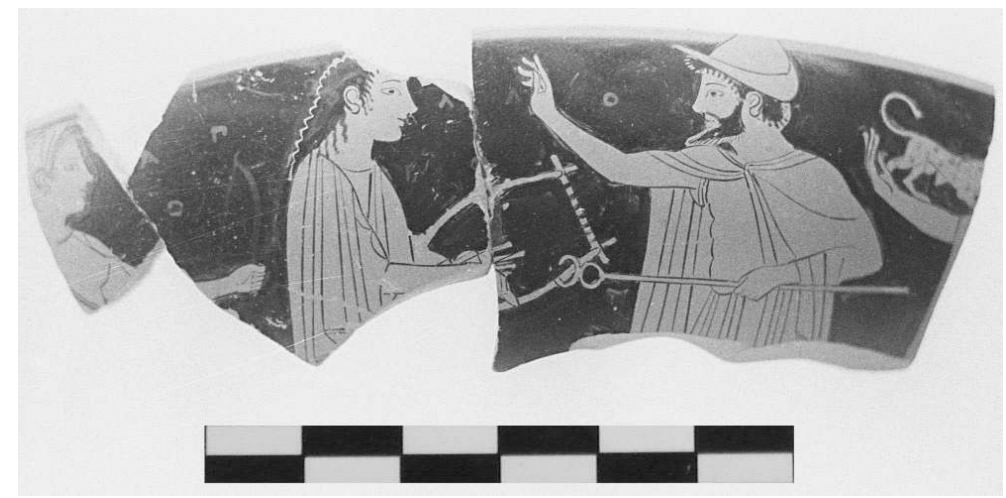

Fig. 3

Le caractère votif des petits objets et des vases est moins évident. Celui des vases en céramique l'est clairement dans certains cas, même en l'absence de dédicace, comme l'attestent plusieurs fois des trous de suspension, notamment pour les plats orientalisants, déjà mentionnés, mais aussi pour des plats attiques à figures noires ${ }^{14}$. Le cas des lékanès thasiennes, très nombreuses, est intéressant. A. Coulié a pu en effet constater que leur décor est souvent «à l'envers » lorsque le vase repose sur son pied, d'ailleurs souvent décoré ${ }^{15}$ : on peut en déduire que ces lékanès étaient directement conçues pour un usage votif et qu'elles portaient leur décor pour qu'il soit parfaitement vu une fois le vase suspendu au mur ${ }^{16}$.

13 Pour des généralités synthétiques relatives aux offrandes dans le monde grec antique, on se reportera désormais commodément à J. BoArdman, T. MANnACK, C. WAGner, B. Forsen, R. PARker, E. VikelA, s.v. «Dedications », ThesCRA I (2004), p. 269-318 (avec une abondante bibliographie).

14 Voir par exemple celui illustré dans MAFFre, TiCHIT, WALTER, l.c. (n. 10), p. 197, fig. 17.

15 Coulié, o.c. (n. 8), p. 144-148.

16 Coulié, ibid., p. 147-148. 
L'usage des vases à boire, très nombreux à l'Artémision, est plus ambigu, en l'absence de contexte clair. Mais une partie au moins des coupes et skyphoi semble bien avoir été votive, comme le montrent quelques tessons porteurs de depinto ou de graffiti. Un très beau fragment de coupe attique à figures rouges (Fig. 3), que J.-J. Maffre a attribué à Épictétos, témoigne de la possible dédicace de vases d'une très grande qualité dans le sanctuaire. On lit sur ce fragment l'inscription peinte $\mathrm{A} \Pi \mathrm{O} \Lambda \Lambda \mathrm{O} \Delta \Omega \Pi \mathrm{O} \Sigma \mathrm{O} \Delta \mathrm{I} \Phi \mathrm{I} \Lambda \mathrm{O} \mathrm{AN}[\ldots$, que l'on est

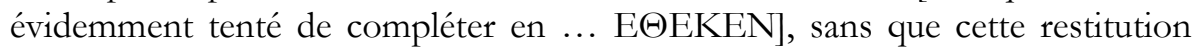
puisse toutefois être tenue pour assurée ${ }^{17}$.

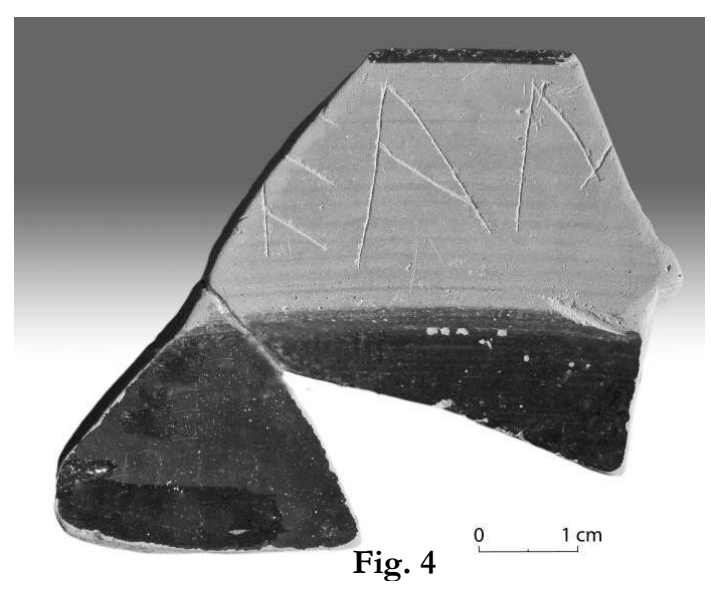

Deux fragments appartenant à une même coupe de Siana portent chacun un graffito qui pourrait être une partie d'une dédicace : on lit sur l'un d'eux (Fig. 4) ${ }^{18}$ la séquence ...]EAN[..., qu'il est tentant de considérer comme la finale d'un nom féminin suivie du début de la forme verbale ANE@EKE, d'autant plus que sur l'autre fragment sont gravées, semble-t-il, les lettres $\Theta \mathrm{EK}^{19}$, à vrai dire peut-être précédées d'un $\mathrm{O}$, ce qui complique toute tentative d'interprétation. Plusieurs fragments de coupes archaïques, enfin, trouvés dans les années 1958-1962 et 1975-1985, portent la probable formule de consécration IPH (Fig. 5) ${ }^{20}$, et il en est de même pour un fragment découvert dans le secteur des Propylées ${ }^{21}$.

17 L'inscription est peinte : cela pose des problèmes qu'il serait trop long d'examiner ici. Cf. MAFFre 1979 (n. 7), p. 63-69; ID. 1988 (n. 7), p. 379-380, fig. 1. L'attribution à Épictétos a été confirmée par D. PAlÉOThodoros, 'pictétos, Louvain et al., 2004 (Coll. d' tudes classiques, 18), p. $20,21,61,72,75,118,153$ et pl. 14,2 .

${ }^{18}$ Fragment de coupe de Siana, inv. 75.A.489 $\alpha$, classé par H. Brijder comme «rappelant le Peintre des Vendanges: Peintres de Naples 81150 et SA 268 » BRIjDer 2000 (n. 11), p. 725, Add. $n^{\circ} 2+$ pl. 248 b-c (fragment du centre).

19 BRIjDER, ibid., pl. 248 b-c à g. (fragment inv. 75.A.489ß). Curieusement, H. Brijder ne dit rien du graffito EAN incisé sur le fragment 75.A.489 $\alpha$ (pl. 248b-c au centre) bien que ce graffito soit visible sur la photo. D'autres interprétations sont toutefois évidemment possibles: la séquence EAN pourrait provenir d'un nom comme $\mathrm{K} \Lambda] \mathrm{EAN}[\Delta \mathrm{PO} \Sigma$. On peut aussi songer à une formule du type de celles où le vase est censé parler lui-même : un tel (ou une telle) m'a

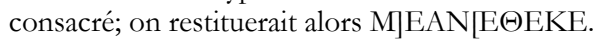

${ }^{20}$ L'interprétation de cette formule a pu être discutée : A. MAZARAKIs Ainian, «Réflexions préliminaires sur les systèmes votifs aux sanctuaires de Kythnos », in Kernos, Suppl. 23 (n. 1), p. 287-318, écrit, avec beaucoup de prudence: «Certains vases à boire à vernis noir portent l'inscription IEPA, ce qui indique peut-être qu'il s'agit d'ustensiles du sanctuaire » (p. 290). Au 
Ces quelques certitudes et présomptions suffisent-elles pour affirmer que toute la céramique trouvée à l'Artémision de Thasos est votive? Certainement pas, car l'énorme quantité des vases mis au jour, si l'on prend en compte toutes les catégories représentées, impose une grande prudence. Certains de ces vases ont dû faire partie

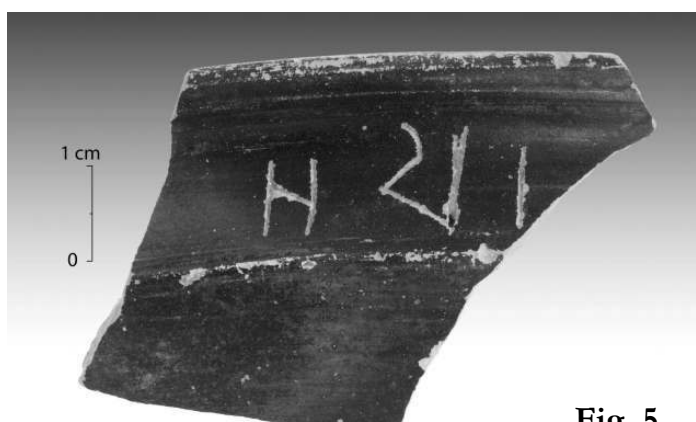

Fig. 5 de la vaisselle nécessaire à la vie quotidienne du sanctuaire, d'autres ont pu être liés à des pratiques cultuelles, comme ont dû l'être les nombreuses lampes mises au jour, sans être pour autant consacrés à la déesse. Quoi qu'il en soit, il parait sûr que bien des vases étaient votifs, et il sera donc légitime, à notre avis, de considérer beaucoup d'entre eux comme offerts à Artémis² ${ }^{22}$.

\section{Les catégories d'objets votifs}

\subsection{Les objets liés à l'activité du tissage : épinétra et pesons}

On trouve en quantité significative des pesons et des épinétra, qui comptent parmi les instruments de l'activité essentielle de la jeune fille et de l'épouse : le tissage. Il y a dans le matériel de l'Artémision de très nombreux pesons, inédits, de formes et d'époques diverses : ronds, coniques ou plutôt tronconiques (Fig. 6). Aucun

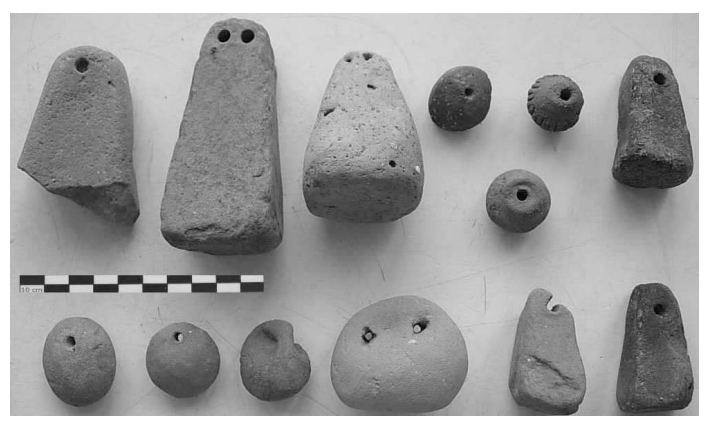

Fig. 6

contraire, PARKER, l.c. (n. 13), p. 274-278, classe sans hésiter les inscriptions du type í@óv parmi les « formulae of dedication ».

21 A. JaCQuemin, BCH 106 (1982), p. 667, fig. 39.

22 Pour une réflexion brève, mais riche et nuancée, sur le caractère des vases trouvés dans un sanctuaire, voir V. STISSI, «From Catalogue to Cultural Context: Bringing Life to Greek Sanctuary Pottery ", in B. SCHMALTZ, M. SÖLDNER (éds), Griechische Keramike im kulturellen Kontext (Akten des Internationalen Vasen-Symposions in Kiel vom 24. bis 28. 9. 2001, veranstaltet durch das Archäologische Institut der Christian-Albrechts-Universität qu Kiel), Münster, 2003, p. 77-79 + pl. 12 : l'auteur estime en dernière analyse que ces vases, plutôt que de jouer un rôle pratique de conteneurs de boissons ou de denrées, étaient parfois liés au service du culte, mais le plus souvent dédiés pour eux-mêmes, sans valeur fonctionnelle, à l'instar des figurines en bronze ou en terre cuite, dans l'idée que l'offrande plairait aux dieux. 
ne porte de dédicace, mais on peut raisonnablement penser qu'ils avaient été offerts à Artémis par des jeunes filles ou des femmes de la cité, comme c'est le cas dans la plupart de ses sanctuaires.
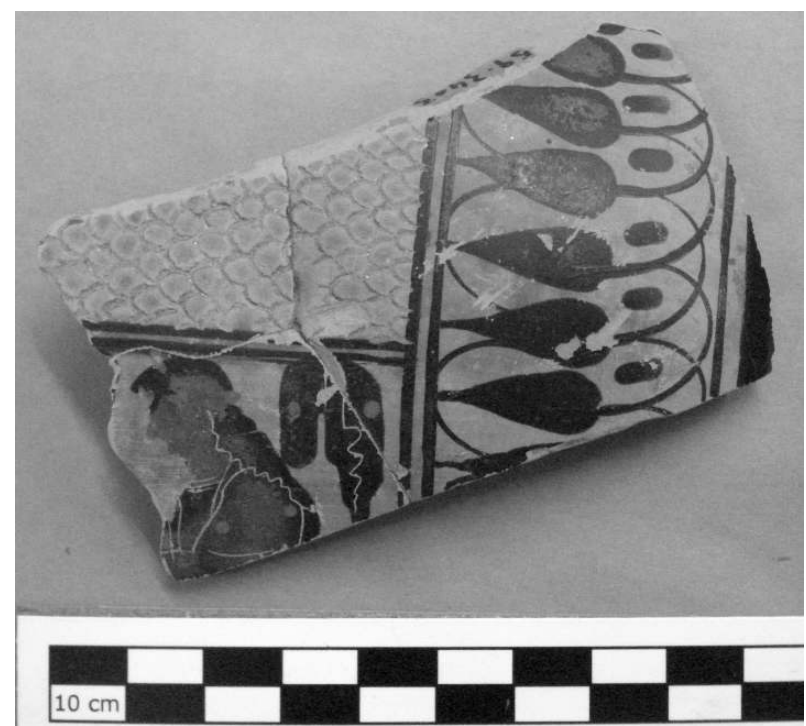

Fig. 7

En quantité plus modeste, une vingtaine de fragments d'épinétra proviennent de plusieurs de ces objets réservés à l'usage féminin ${ }^{23}$. D'une façon générale, d'après les indications fournies par Ch. Mercati24, 75\% des épinétra à figures noires et $50 \%$ de ceux à figures rouges dont la provenance est connue ont été trouvés dans des sanctuaires de divinités féminines : Athéna (Acropole d'Athènes : 23 à figures noires; Lindos, sanctuaire d'Athéna Lindia : 1 à figures noires - 1 sans décor figuré), Déméter (sanctuaires d'Éleusis : 4 à figures noires; de Corinthe : 4 à figures noires; de Cyrène : 3 à figures noires; de Sélinonte : 2 à figures noires) et surtout Artémis (Brauron, 20 à figures noires 18 à figures rouges; Mounichie : 1 à figures noires -2 à figures rouges -2 sans décor figuré; Amarynthos, en Eubée : 2 à figures noires; et Thasos : 18 à figures noires). L'iconographie pose un problème d'interprétation intéressant. L'un des fragments de Thasos

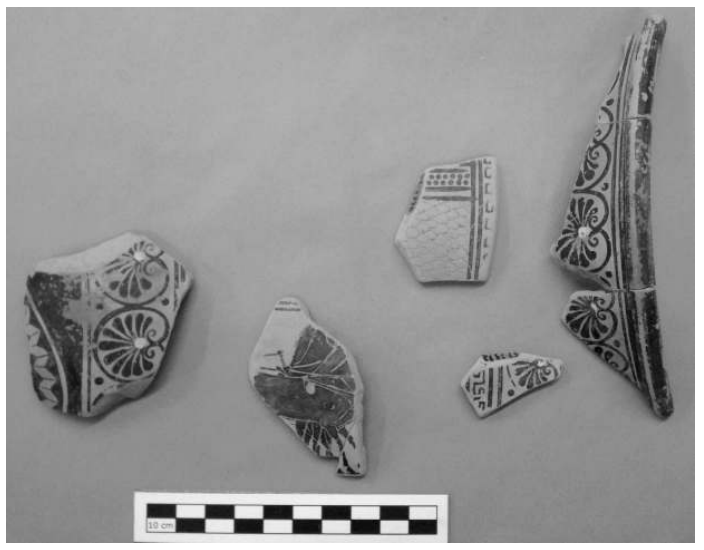

Fig. 8

23 Sur les épinétra, on se reportera aux ouvrages de P. Badinou et Ch. Mercati cités supra, n. 11. Voir aussi F. HeInRICH, Das Epinetron. Aspekte der weiblichen Lebenswelt im Spiegel eines Arbeitsgeräts, Rahden (Westf.), 2006 (Internationale Archäologie, 93) (les fragments de Thasos sont pris en compte et catalogués p. 177-178, nos 99-109), et en dernier lieu S. SAVELLI, "La vergine e l'epinetron: la tomba 44 della necropoli di età classica ad Efestia (Lemno) », AnnuarioS AIA 84 (2006) [2008], p. 359-380.

24 Mercati, o.c. (n. 11). 
présente une scène qui convient parfaitement dans le mundus muliebris : on y voit des femmes et des tissus (Fig. 7); les autres offrent des images moins "féminines », avec des éléments liés aux occupations masculines: des quadriges (Fig. 8)25. Certains y ont vu un désir de réunir les sphères des deux sexes. P. Badinou, par exemple, considère que « la plupart des épinétra ont été trouvés dans des sanctuaires. Cela pourrait signifier qu'ils ont été commandés pour servir directement d'ex-voto. Offrandes pieuses, ils témoignent d'un oikos équilibré entre travaux féminins au gynécée (l'épinétron) et activités masculines sportives et violentes à l'extérieur (l'imagerie) $»^{26}$.

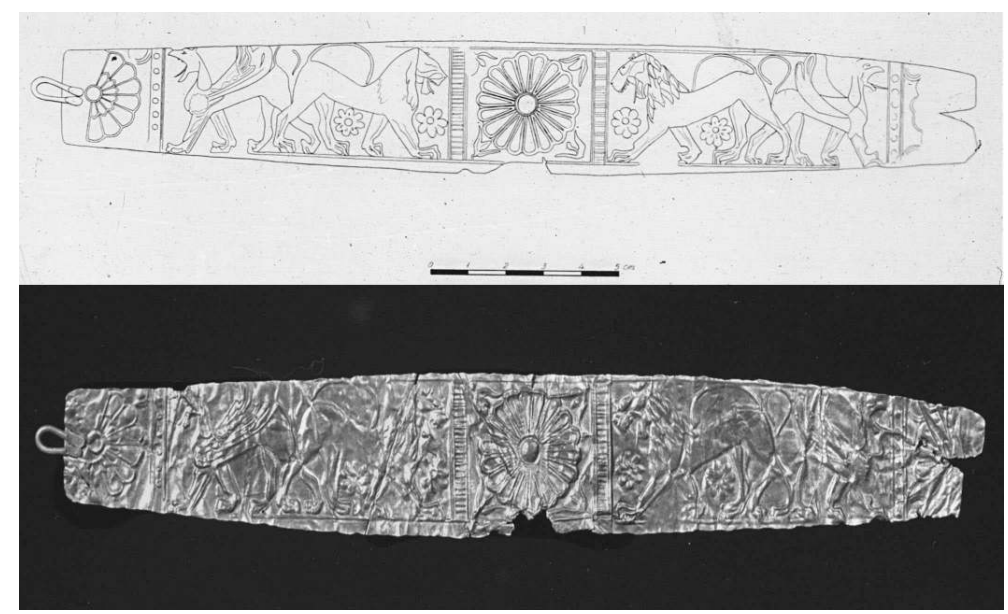

Fig. 9

\subsection{Les éléments de parure}

Les bijoux recueillis sont assez peu nombreux ${ }^{27}$. On a trouvé à l'Artémision de Thasos des éléments de parure en bronze - boucles d'oreille, bracelets, fibules -, des boucles d'oreille et des anneaux en argent, ainsi que quelques rares éléments de parure en or. Une bague à chaton en or aplati en forme de losange porte les traces d'un décor qui pourrait être une tête de lion ou de satyre ou encore un gorgoneion ${ }^{28}$. On a exhumé également des perles creuses constituées de deux moitiés pyramidantes soudées entre elle. La pièce la plus remarquable est un diadème en or, d’origine rhodienne, décoré de motifs en

25 Sans parler ici du problème de la restauration de l'un des épinétra de Thasos avec une tête de lion plastique qui n'est pas certaine et qui n’a pas de sens évident sur un épinétron.

26 BADinOU, o.c. (n. 11), p. 50.

${ }^{27}$ Nous remercions Cl. Prêtre, qui a eu l'obligeance de nous faire part de ses recherches sur le petit matériel votif du sanctuaire et dont la publication devrait paraitre prochainement dans la collection des't $t$ Thas.

28 BCH 102 (1978), p. 827, fig. 34. 
repoussé : une grande rosette au centre, encadrée de deux lions tournés vers le centre et de deux griffons tournés vers les extrémités; dans le champ, des rosettes; aux extrémités, deux demi-rosaces (Fig. 9)29.

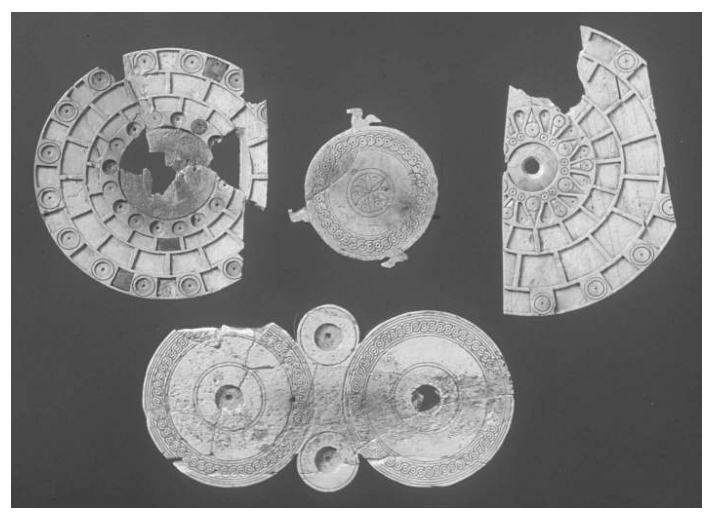

Fig. 10

Les éléments de parures sont parfois en ivoire ou en os (Fig. 10). On a mis au jour à l'Artémision de Thasos des fibules bilobées d'une grande qualité. Ce sont là des offrandes courantes dans les sanctuaires de divinités féminines à l'époque archaïque. Certaines, très proches des exemplaires de Thasos, ont été offertes à l'Artémision d'Éphèse ${ }^{30}$ ou dans le sanctuaire d'Artémis Orthia de Sparte ${ }^{31}$. Artémis n'a cependant pas l'exclusivité de ce genre d'objet votif.

En relation avec ces parures, des miroirs de bronze sont aussi présents, d'un type simple ou plus complexe, comme l'atteste le support en bronze, de fabrication péloponnésienne, daté du milieu du VI e siècle av. J.-C. et représentant une jeune femme drapée, debout sur une base carrée, les bras au-dessus de la tête, comme pour soutenir le disque du miroir, qui manque (Fig. 11) 32 .

\subsection{Grande et petite plastique de terre cuite}

Le sanctuaire thasien avait reçu, entre autres ex-voto remarquables, quelques grandes statues en terre cuite

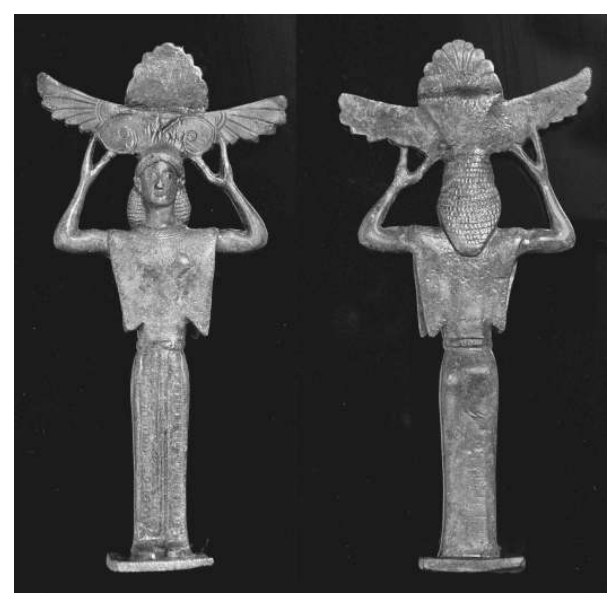

Fig. 11

29 Guide de Thasos (2000), p. 297, fig. 259.

30 Sur les petits objets en ivoire de l'Artémision d'Éphèse, voir par exemple D.-G. HoGARTH, Excavations at Ephesus : the Archaic Artemisia, Londres, 1908, p. 186-198, pl. XXXII-XLII.

31 R. MCGillivray Dawkins, The Sanctuary of Artemis Orthia at Sparta, Londres, 1929, p. 224 225, pl. CXXXII.

32 BCH 93 (1969), pl. XXI-XXII; Guide de Thasos (2000), p. 297 et 299, fig. 261. 
d'époque archaïque ${ }^{33}$. Le coût moindre de la matière première n'enlève rien au travail expérimenté des artisans auteurs de ces œuvres qui présentent les mêmes types que la grande plastique de marbre contemporaine, tout comme la petite plastique de terre cuite. Parmi les nombreux fragments, ceux d'un buste féminin appartiennent à une statue plus grande que nature (on estime sa hauteur à 1,90 m). De production locale, alliant moulage et modelage, elle possède tous les caractères du style dédalique du VIIe siècle (Fig. 12).

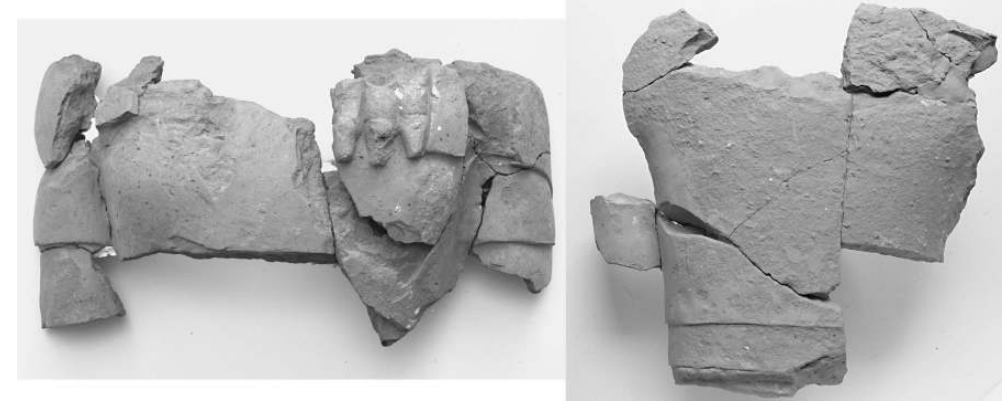

Fig. 12

Figurines et protomés de terre cuite font partie des offrandes courantes dans les sanctuaires de divinités féminines. À Thasos, ce sont l'Artémision et le Thesmophorion qui en ont donné le plus grand nombre: environ 25000 fragments (dont entre la moitié et les deux tiers de protomés) rien qu'à l'Artémision ${ }^{34}$ ! Plus modestes par leurs dimensions mais bien plus nombreuses que les statues, elles couvrent, pour l'Artémision de Thasos, la période allant du $\mathrm{VII}^{\mathrm{e}}$ siècle à la période hellénistique ${ }^{35}$. Malgré une certaine variété, ce sont les types féminins qui, comme dans la plupart des sanctuaires consacrés à des déesses, sont très largement majoritaires ${ }^{36}$, notamment dans la seconde moitié

33 BCH 85 (1961), p. 927-928, fig. 25-27. Le texte parle d'au moins douze statues de korés différentes. Certaines de ces statues ont été mentionnées dans les Chroniques des fouilles du BCH, d'autres (ou les mêmes) présentées par N. Weill dans son ouvrage sur la plastique archaïque de Thasos (' tThas, XI : 1985); beaucoup sont inédites et en cours d'étude par A. Muller.

${ }^{34}$ Ce sont les chiffres donnés par A. MulLER, «Le tout ou la partie. Encore les protomés: dédicataires ou dédicantes ? ", Kernos, Suppl. 23 (n. 1), p. 83.

${ }^{35}$ L'ensemble est actuellement en cours d'étude par une équipe d'Halma-Ipel - UMR 8164; $c$. A. Muller et al., "Thasos : les terres cuites votives de l'Artémision», BCH 120 (1996), p. 891900; EID., « Recherches sur la coroplathie thasienne : campagnes d'étude 1996-2002 », BCH 126 (2002), p. 231-247.

36 On note la présence de quelques kouroi ou de figures de banqueteurs allongés, mais en proportion bien inférieure, de même que l'on a trouvé à Brauron quelques statuettes de jeunes garçons : voir B. LunDGREEN, "Boys at Brauron. The significance of a votive offering", in T. Fisher-Hansen, B. Poulsen (éds), From Artemis to Diana, The Goddess of Man and Beast, Copenhague, 2009 (Acta Hyperborea 12), p. 117-126, et V. Mitsopoulos LeON, BPAYPQN. Die Tonstatuetten aus dem Heiligtum der Artemis Brauronia. Die frühen Statuetten (7. bis 5. Jh. v. Chr.), Athènes, 2009 (Bibliothek, der Archäologischen Gesellschaft zu Athen, 263). 
du VIe siècle : femmes trônant, coiffées d'une couronne ou d'un bandeau qui maintient un voile; korés debout, en chiton et himation, tenant un objet dans la main. Les protomés, que l'on considérerait volontiers comme des versions tronquées des figurines complètes ${ }^{37}$, présentent elles aussi les types féminins les plus courants.

On a découvert en outre un certain nombre de figurines animales en terre cuite. Selon les termes de la Chronique du BCH de 1958, « un bestiaire varié a été rassemblé : oiseaux, béliers, singes, sangliers et porcs, ours, tortues, serpent $»^{38}$. Certains de ces animaux sont de production corinthienne (Fig. 13); d'autres sont

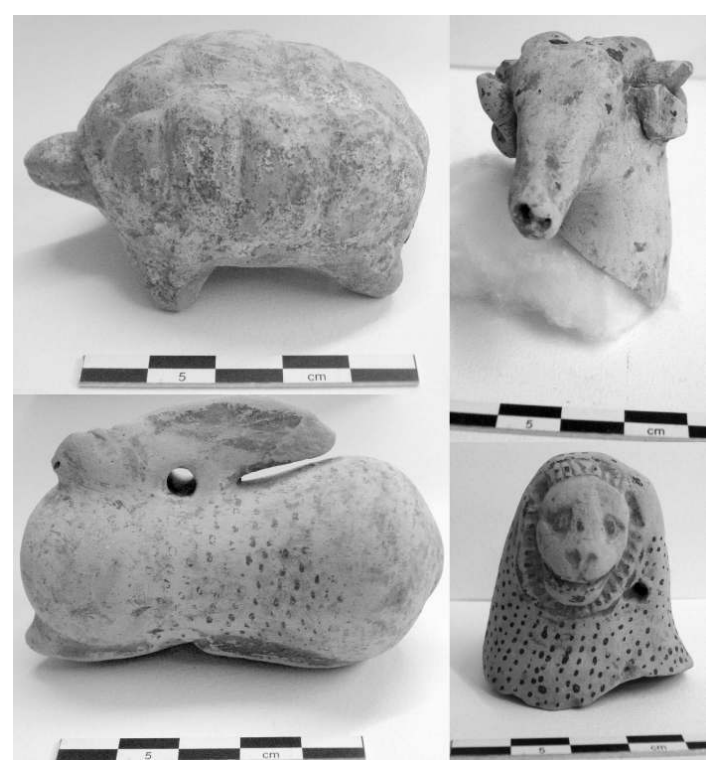

Fig. 13 difficiles à caractériser. On peut ajouter à la liste des lions et des panthères ${ }^{39}$, dont la présence est récurrente dans tous les types d'offrandes (vases, bijoux, ivoires...).

Une catégorie de figurines de terre cuite de l'époque archaïque parait plus originale et peut-être représentative du culte voué à l'Artémis de Thasos: il s'agit des figurines dites de la «Dame au Polos» (Fig. 14). Cette série particulière est presque exclusivement présente à Thasos, dans le sanctuaire d'Artémis ${ }^{40}$. Trois générations en furent produites par les ateliers thasiens dans la $2^{\mathrm{e}}$ moitié du VIe siècle, à partir d'un modèle créé dans le $2^{\mathrm{e}}$ quart de ce même siècle. Malgré les trois variantes attestées, les caractéristiques sont les mêmes tout au long de la production : femme trônant, le visage souriant, coiffée d'un

\footnotetext{
${ }^{37}$ Nous suivons sur ce point l'opinion d'A. Muller, exprimée notamment dans l'article récent cité supra (n. 34), p. 81-95.

38 BCH 82 (1958), p. 810.

39 Voir aussi le vase plastique corinthien publié dans 't Thas XI (1985), p. 133, pl. 32, n 123; pour un catalogue des figurines animales archaïques, voir 't thas XXI (2009), p. 545-559.

40 On la rencontre, en très petit nombre, dans les autres sanctuaires de Thasos et de sa Pérée (Néapolis-Kavala, Oisymé). Le type ne se trouve pas en dehors de cet espace. Pour ce qui est des 51 fragments recueillis dans les fouilles anciennes, voir 't Thas XI (1985), p. 147-220; pour une analyse plus récente prenant en compte les trouvailles nouvelles, voir St. HUYSECOM-HAXHI, « La Dame au Polos thasienne : établissement d'une série ", in A. MulLer (éd.), Le moulage de terre cuite dans l'Antiquité : création et production dérivée, fabrication et diffusion (Actes du XVIIIe colloque du Centre de Recherche Archéologique - Lille III, 7-8 déc. 1995), Lille, 1997, p. 155-180; EAD., 'tThas XXI (2009), p. 342-356.
} 
haut polos d'où descendent deux mèches parotides avec boucles en perles. $\mathrm{N}$. Weill voyait dans « la dignité de l'attitude et de l'expression, [ainsi que dans] la permanence absolue des particularités typologiques » le signe d'une possible reproduction en réduction d'une statue de culte, qui n'a toutefois pas été retrouvée, tout en appelant elle-même à la prudence face à cette hypothèse, faute d'élément probant ${ }^{41}$.

S'il est vrai que cette interprétation, très tentante, rendrait compte de l'originalité de ces statuettes et aurait le mérite d'expliquer que quelques-unes des caractéristiques iconographiques aient été reprises par la suite sur des figurines au type plus banal de la koinè ionienne, aucun attribut ne permet toutefois l'identification certaine de la déesse. Il faut d'ailleurs reconnaitre que si le style est parfaitement original, le type, lui, de la dame trônant et portant polos ou diadème, ne l'est pas. D'autres hypothèses ont été émises,

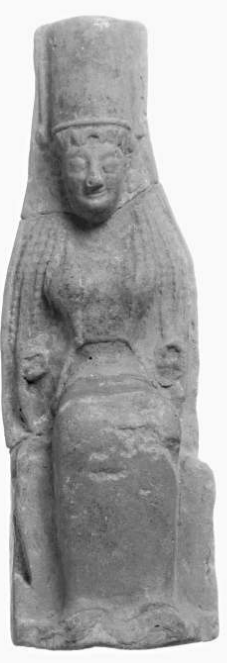

Fig. 14 comme la représentation de prêtresses ou plus simplement de fidèles ${ }^{42}$.

\subsection{Les vases}

On recense à l'Artémision de Thasos quelques vases de bronze, qui restent très rares. En revanche, plus encore que les figurines, les vases de terre cuite constituent la catégorie d'objets la plus nombreuse, avec une très grande variété d'origines, de techniques et de formes: céramique à décor figuré de divers ateliers - Cyclades, Grèce de l'Est, Chios, Corinthe, Athènes ${ }^{43}$, Sparte et Thasos

41 WeILL, l.c. (n. 12), p. 142.

42 St. Huysecom, A. Muller, «Déesses et/ou mortelles dans la plastique de terre cuite. Réponses actuelles à une question ancienne », Pallas 75 (2007), p. 240. Sur les prêtresses et leurs représentations, voir en dernier lieu J.B. Connelly, Portrait of a Priestess: Women and Ritual in Ancient Greece, Princeton, 2007.

${ }^{43}$ Sur les trouvailles de céramique attique, qui proviennent essentiellement de l'Artémision, voir supra, n. 7, 10 et 17; outre les articles signalés dans ces notes, l'un d'entre nous a sous presse deux études particulières: J.-J. MAFFRE, «Fragments de coupes à lèvre attiques trouvés à Thasos", in Mélanges H. Sarian (à paraître en 2011); ID., "Remarques préliminaires sur la céramique attique à figures rouges trouvée à Thasos depuis 1956 », in Hommage à Marina Sgourou (à paraître en 2011). 
même -, céramique à vernis noir, céramique à reliefs, céramique commune. Quelques vases plastiques de forme animale, d'origine corinthienne ou attique, rappellent le répertoire animal des figurines de terre cuite.

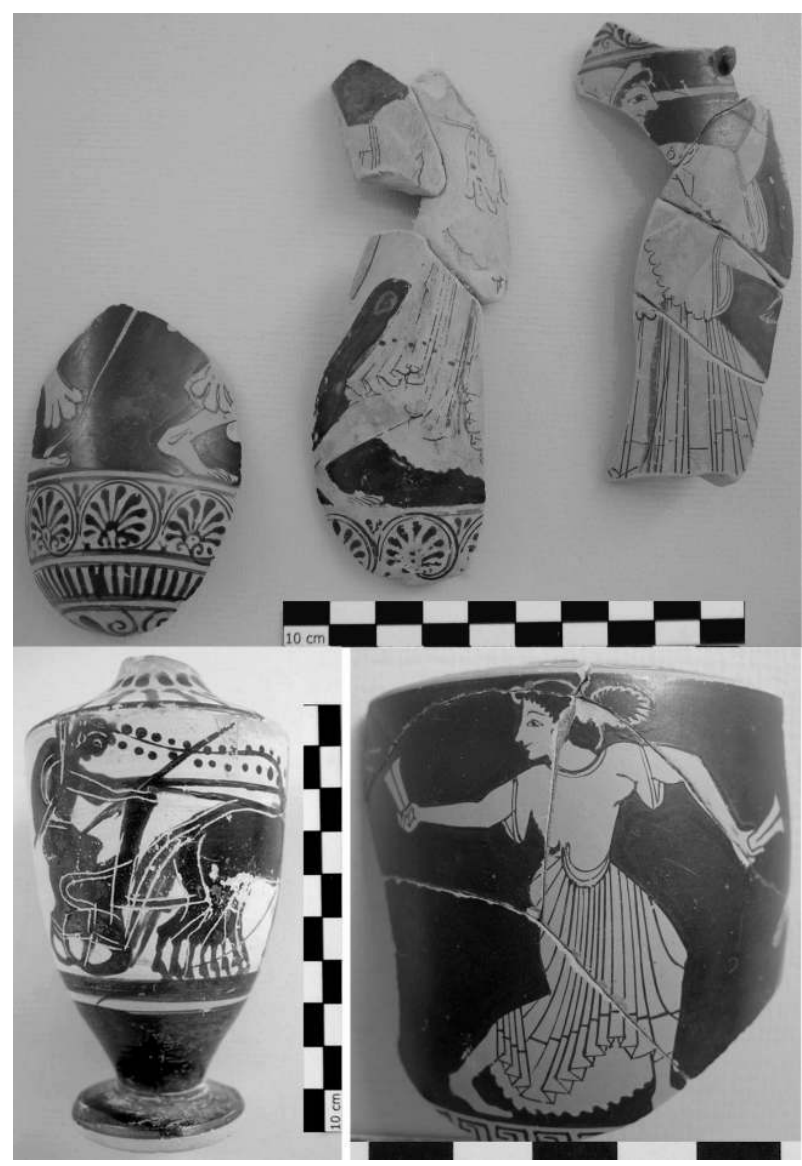

Fig. 15

Les plats de style orientalisant de divers ateliers du VII ${ }^{e}$ siècle sont relativement nombreux. Les ateliers thasiens montrent aussi une certaine prédilection pour cette forme, même si elle n'est pas la plus courante, et on trouve leur production en nombre à l'Artémision. Parmi les pièces les plus remarquables, le célèbre plat au Bellérophon présente, quant à lui, une sorte de scène de chasse, et le décorateur de ce plat n'est autre que celui de l'amphore de Délos qui porte sur son col une Potnia aux lions. Quelques phiales à décor surpeint, apparemment attiques, témoignent de libations. Il faut peutêtre envisager le même usage pour les nombreuses lékanès thasiennes à figures noires, qui constituent la forme privilégiée par les ateliers thasiens. Les études du matériel des autres sanctuaires, toujours en cours, ne permettent pas encore de dire si ces lékanès ont été produites essentiellement pour être dédiées dans le sanctuaire d'Artémis. Les vases à parfum sont assez peu nombreux. On compte quelques fioles en verre et pâte de verre, ainsi que quelques dizaines de pièces en argile : lécythes attiques, aryballes et alabastres corinthiens, alabastre attique à figures rouges représentant une ménade en course vers la droite, peut-être suivie de Dionysos portant une peau de panthère (Fig. 15). 


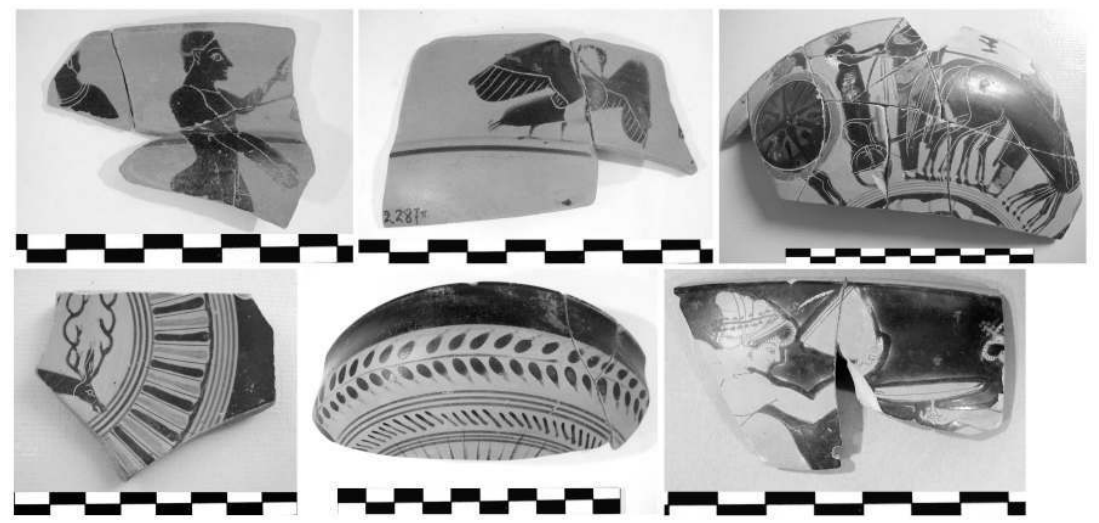

Fig. 16

La catégorie de vases la plus fournie est constituée par d'innombrables vases à boire : coupes (Fig. 16) et skyphoi. Le même phénomène se retrouve par exemple à l'Artémision d'Éphèse, où le matériel céramique compte, d'après des estimations récentes, environ 50\% de vases à boire, ce qui pourrait répondre à des pratiques cultuelles comprenant des symposia ${ }^{44}$. Les vases laconiens sont très rares : une centaine de fragments de coupes a été répertoriée pour le moment. En ce qui concerne les séries attiques ${ }^{45}$, on a identifié environ 40 fragments de coupes des Cômastes, 600 de Siana, 400 de coupes à lèvre, plusieurs milliers de fragments de coupes à bandes (si l'on inclut les coupes à palmettes), plus de 1000 de coupes de Droop, environ 1500 de coupes de Cassel, plus de 1000 de coupes à vasque hémisphérique (coupes à yeux, coupes du style "prolongation»), ainsi qu'une centaine de fragments de coupes à figures rouges. L'inventaire des fragments de skyphoi attiques à figures noires n'est pas achevé, mais il est sûr que leur nombre dépasse largement les 2000 exemplaires; ceux à figures rouges sont en revanche très rares. Par comparaison, pour la figure noire attique, on n'a qu'une centaine de fragments de lékanès ${ }^{46}$ (bien moins nombreux que ceux des lékanès thasiennes), une vingtaine de fragments de plats (mais beaucoup plus de fragments de plats orientalisants et thasiens à figures noires), une dizaine de fragments de cratères, une centaine de fragments de lécythes, olpès, œnochoès, de rares fragments d'amphores, hydries, alabastres, phiales.

44 M. Kerschner, "Keramik aus dem Heiligtum des Artemis », in U. Muss (éd.), Die Archäologie der ephesischen Artemis. Gestalt und Ritual eines Heiligtums, Vienne, 2008, p. 125-133. Cet ouvrage apporte par ailleurs, notamment dans la douzaine de contributions regroupées dans la partie intitulée Archäologie und Ritual (p. 79-200), d'intéressantes considérations sur les offrandes faites à l'Artémis d'Éphèse.

45 Nous espérons pouvoir donner un aperçu plus précis d'une partie des coupes attiques archä̈ques mises au jour à l'Artémision dans un prochain fascicule des 't Thas.

46 Presque toutes attribuables au Peintre du Polos; quelques-unes sont signalées dans MAFFrE 1990 (n. 7), p. 410-411, nos 1-4 et p. 419, fig. 1-4. 


\subsection{Les objets précieux ${ }^{47}$}

Un certain nombre d'offrandes en ivoire finement travaillé sont certainement des éléments de décor de coffres ou de meubles précieux. On a notamment découvert des disques en ivoire d'éléphant. De dimensions variables, richement ouvragés, ils portent parfois encore des traces d'incrustation de matière précieuse, comme de l'ambre. La fonction exacte de ces disques n'est pas assurée. L'absence de traces au dos oblige à réfuter l'hypothèse de bijoux, d'un type proche de celui de la fibule. Il s'agit à n'en pas douter d'éléments d'ornementation, mais peut-être moins de parures que de meubles ou coffres précieux. Une plaquette d'ivoire ciselée, reproduisant l'image d'une jeune fille drapée, est sans doute aussi un décor appliqué de coffre ou de meuble précieux. Le travail est très précis et les détails sont soigneusement gravés, sur cette plaque très fine, haute d'à peine $4,5 \mathrm{~cm}$ et épaisse de 0,2 cm (Fig. 17). Mise au

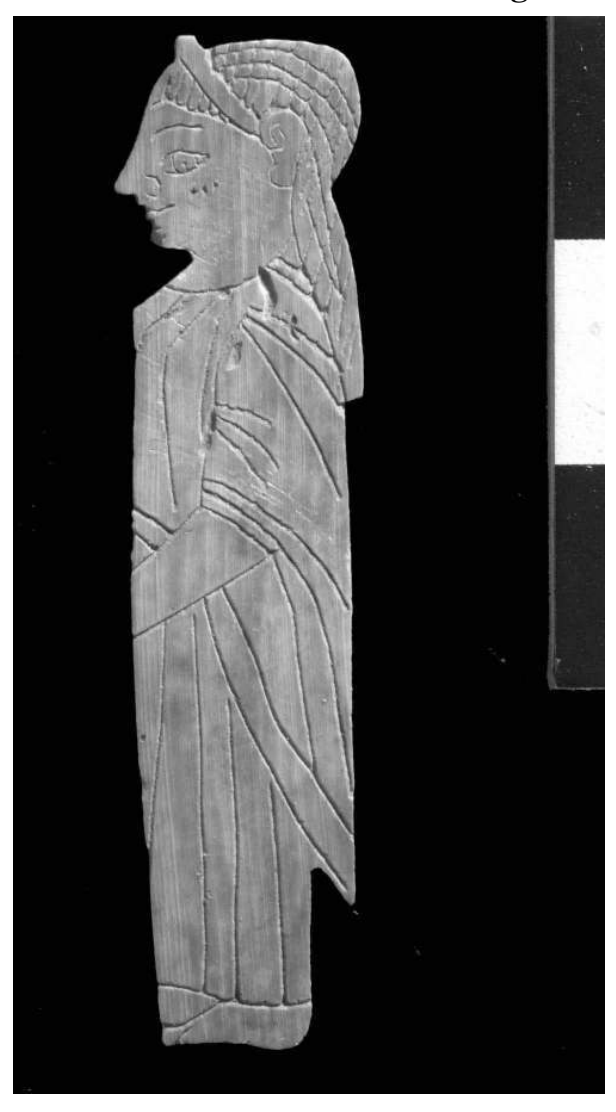
jour dans les remblais du péribole carré (terrasse supérieure), elle doit dater de la fin de la période archaïque.

Une protomé de chevaux attelés formant un bige, en ivoire d'éléphant ${ }^{48}$, est aussi à n'en pas douter un élément d'applique de meuble précieux. On a souvent constaté dans les autres sanctuaires d'Artémis des liens entre Artémis et les chevaux, sans que l'on propose toutefois une explication convaincante à cette éventuelle relation. On a parfois souligné le lien entre le Soleil et le culte d'Artémis $^{49}$ : les figures de chevaux pourraient alors représenter le char d'Hélios. Mais aucune autre offrande, à notre connaissance, dans l'Artémision de Thasos, ne paraît liée à un culte d'Hélios. Même si la figure du cheval rappelle une interpréta-

\footnotetext{
47 On regroupe dans cette catégorie, il est vrai un peu vague, des petits objets votifs rares, souvent d'une grande valeur.

48 Chronique des fouilles 1959, BCH 84 (1960), p. 862, fig. 3.

49 A. Bammer, « Kosmologische Aspekte der Artemisionfunde », in U. Muss (éd.), Der Kosmos der Artemis von Ephesos, Vienne, 2001, p. 16-17.
} 
tion possible de l'épiclèse "Pôlô» de l'Artémis thasienne, le meuble auquel appartenait la protomé était avant tout, à notre avis, un objet précieux offert à la déesse, tout comme d'autres meubles ornés, notamment, de belles têtes de lions.

Le matériel votif de l'Artémision thasien possède sans doute le plus grand nombre de boutons-bobines en ivoire incrusté d'ambre ou en cristal de roche recueillis dans un même sanctuaire. Or ce type d'objets est rare ailleurs, et seul le sanctuaire d'Artémis à Ephèse semble avoir reçu des offrandes similaires en nombre important. On connait aussi des objets de même forme (mais avec un décor différent) au sanctuaire d'Artémis Orthia de Sparte. Il s'agit peut-être d'un objet votif plus volontiers offert à Artémis et ainsi d'un élément d'identification possible d'un sanctuaire dédié à cette déesse. Malheureusement, les études publiées à ce jour sont encore trop incomplètes pour que l'on puisse présenter sur ce point des conclusions fermes, d'autant que l'on ignore encore la fonction réelle de tels objets.

On mentionnera enfin la découverte d'un ou peut-être plusieurs œufs d'autruche polis, qui ont été identifiés assez récemment par Fr. Poplin et considérés par lui comme des objets précieux, dignes d'être offerts dans un sanctuaire ${ }^{50}$.

\section{Quelle(s) personnalité(s) d'Artémis ? Quelques éléments d'interprétation}

\subsection{Les représentations d'Artémis}

N. Weill, dans son étude fondamentale sur l'iconographie des offrandes de l'Artémision thasien, estimait que, pour ce sanctuaire, « il faut souligner d'emblée que, dans un répertoire figuré très diversifié, les images caractérisées d'Artémis sont rares, et se rapportent à un petit nombre de thèmes ${ }^{51}$.

Les premières représentations liées à Artémis sont celles de la Potnia Thérôn ailée. On la reconnait sur un plat cycladique fragmentaire de style polychrome du milieu du VII siècle av. J.-C. (Fig. 18), ainsi que sur un plat de la Grèce de l'Est, lui aussi polychrome, de la fin du VII siècle (Fig. 19) : la Potnia, les ailes déployées de chaque côté, est représentée en course vers la droite. N. Weill restitue sur le plat cycladique des fauves dans les mains de la divinité, selon une iconographie bien

\footnotetext{
50 Dans une lettre du 10 octobre 1994, cet éminent chercheur du Museum National d'Histoire Naturelle de Paris, qui a souvent fait bénéficier de sa science zoologique les archéologues de Thasos, écrit : «Il y a de l'œuf d'autruche dans le matériel de l'Artémision... Il s'agit d'une dizaine de petits fragments ... Leur face externe a été polie ... Il ne semble pas que ce polissage ait été remarqué. Il résulte d'un travail soigneux et long, qui fait de ces œufs des objets précieux ». Il résume ces observations dans un article intitulé "Sur le polissage des œufs d'autruche en archéologie », in H. Buitenhuis, H.-P. UerPmann (éds), Archaeozoology of the Near East II, Leiden, 1995, p. 131.

51 N. WeILL, l.c. (n. 12), p. 137-148. La citation est empruntée à la p. 138.
} 
connue $^{52}$. Le plat gréco-oriental la représente tenant des serpents. On retrouve cette figure encore au $\mathrm{VI}^{\mathrm{e}}$ siècle sur des reliefs de terre cuite moulés provenant sans doute des ateliers thasiens ${ }^{33}$ (Fig. 20), au moment où des représentations d'Artémis apparaissent sur quelques vases peints à figures noires.
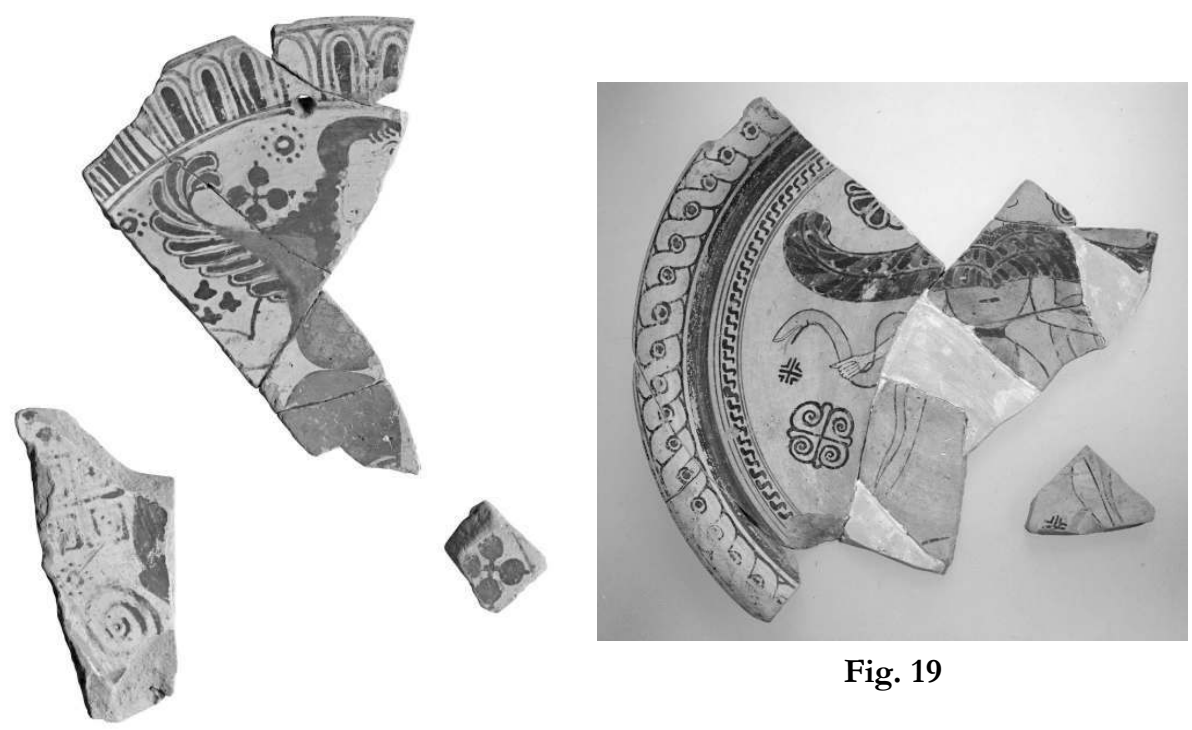

Fig. 19

\section{Fig. 18}

On pourrait être tenté de mettre en rapport avec la Potnia Therôn les très nombreux animaux (quadrupèdes ou oiseaux) qui apparaissent sur beaucoup d'aryballes et alabastres corinthiens, de lékanès thasiennes et attiques, de coupes attiques à lèvre et à bandes, de petits vases (skyphoi, lékanès, couvercles) du Swan group attique ou d'imitation locale ${ }^{54}$, et surtout les célèbres plats orientalisants du VII ${ }^{e}$ siècle. Mais ces représentations sont trop courantes pour qu'on puisse, à notre avis, leur attribuer un tel sens.

${ }^{52}$ L. KAHIL, s.v. «Artemis », LIMC II (1984), nos 21-49 (« la Potnia Thérôn d'époque hellénique identifiée à Artémis », ailée), et N. ICARD-Gianolio, s.v. "Potnia », LIMC VIII, Suppl. (1997), notamment nos 11-30 et 38-40. Les vases de Thasos, peu connus, ne sont pas cités.

53 Ces fragments sont considérés par St. Huysecom-Haxhi comme des représentations de

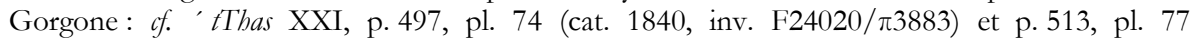
(cat. 1867, inv. F20422/ $\pi 1822+\pi 5643$ ).

54 C. CHABot Aslan, "Swan Imagery at the West Sanctuary at Troia ", in SOMA 2007 Proceedings of the XI Symposium on Mediterranean Archaeology, Istanbul Technical University, 2429/04/2007), Oxford, 2009 (BAR IS, 1900), p. 54-60, pense que l'abondance de fragments de petits vases à figures noires décorés de cygnes dans le sanctuaire Ouest de Troie montre qu'il s'agit sans doute d'un sanctuaire d'Artémis (ou de Cybèle, ou d'Aphrodite). 
En réalité, la divinité du monde sauvage que peut être Artémis est assez peu visible à travers les offrandes de son sanctuaire thasien. Le fragment de la coupe attique à figures rouges attribuée à Épictétos offre tout de même une image de la déesse en rapport avec cet aspect (Fig. 3). On y voit Hermès, Apollon et Artémis, chacun caractérisé par un attribut: Apollon une lyre, Hermès le caducée, Artémis un arc. C'est donc l'Artémis chasseresse qui est ici évoquée.

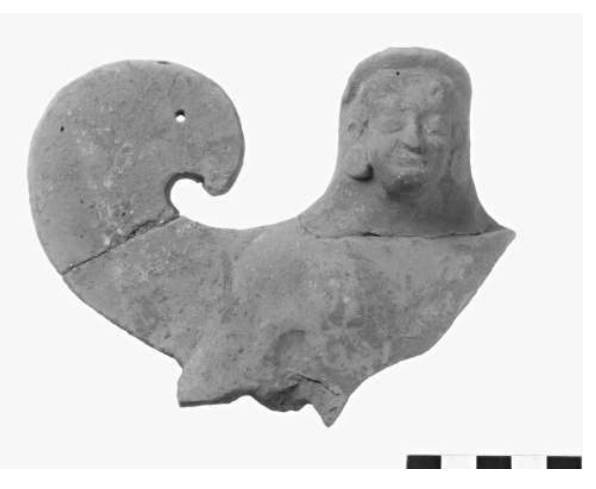

Fig. 20

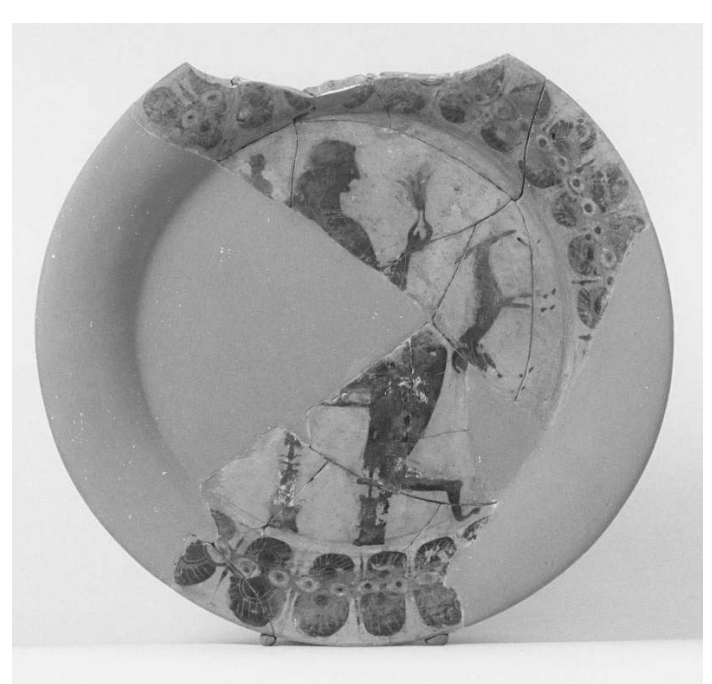

Fig. 21

Le thème iconographique d'Artémis à la biche 55 est attesté au moins deux fois. Sur un grand plat thasien à figures noires datant, selon A. Coulié, $\mathrm{du} 3^{\mathrm{e}}$ quart du $\mathrm{VI}^{\mathrm{e}}$ siècle (Fig. 21) ${ }^{56}$, la déesse est installée sur un trône, les pieds posés sur un tabouret; elle tient à la main une fleur; une petite biche est librement dessinée dans le champ, comme une sorte de motif de remplissage, mais c'est elle qui permet l'identification de la divinité comme Artémis. L'autre représentation de ce thème est fournie par une figurine de terre cuite attique; le type habituel de la péplophore assise est individualisé par la présence de la biche dans les bras de la figure, ainsi identifiable comme Artémis ${ }^{57}$. L'alliance de la fleur et de la biche sur le plat offre peut-être une clé d'interprétation et met en avant un aspect bien connu des fonctions d'Artémis, présent aussi chez la Potnia Thérôn : la fécondité, dont la déesse est garante.

55 Sur ce thème, voir par exemple S. KLINGER, «Artemis Holding a Deer: The Iconography of the Motif on a Red-Figure Oinochoe in Kiel », in SCHMALTZ, SöLDNER, o.c. (n. 22), p. 142-144, pl. 25, 2-4.

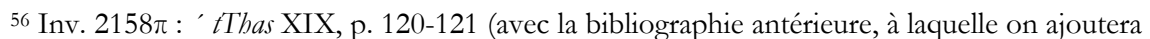
L. KAHIL, LIMC II, s.v. "Artemis ", $\mathrm{n}^{\circ}$ 670; date proposée : fin du VI s.), p. 153-154, fig. 7 , p. 199 et pl. $85, \mathrm{n}^{\circ} 329$.

${ }^{57}$ WeILL, l.c. (n. 12), p. 140, fig.7. 


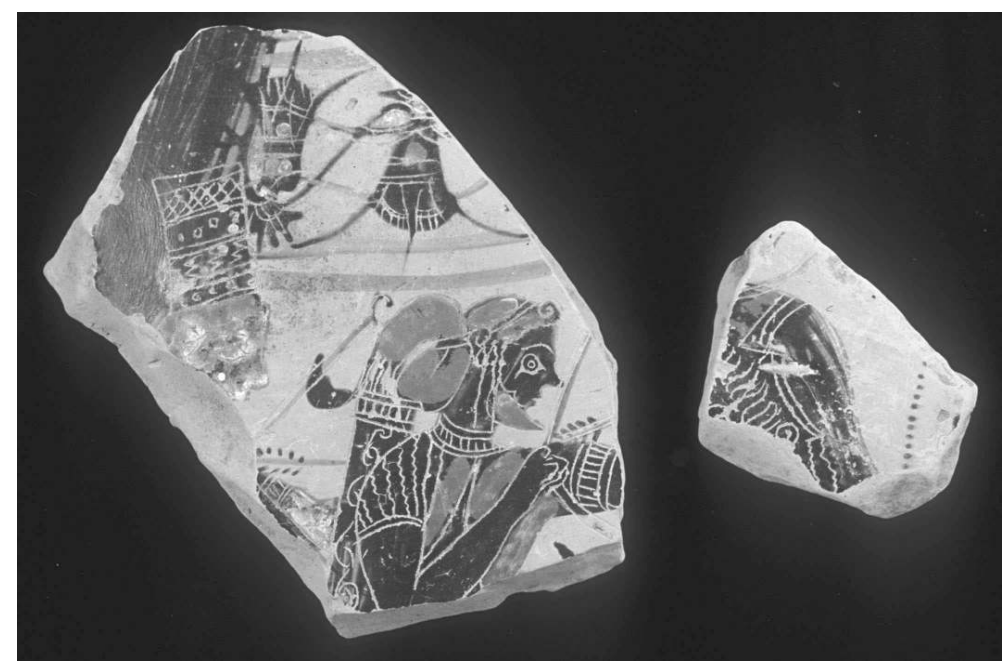

Fig. 22

Deux fragments d'une amphore à figures noires illustrent le meurtre de Tityos (Fig. 22). A. Coulié les considère comme de fabrication thasienne et les date vers 560. Elle attribue le vase à son Peintre de Troïlos ${ }^{58}$. Apollon et, derrière lui, Artémis s'apprêtent, d'un geste identique, à décocher une flèche sur Tityos, dont la tête est conservée sur le second fragment. La déesse porte un haut polos richement décoré de motifs variés, qui évoque le type de la «Dame au Polos» dont nous avons déjà parlé, mais que l'on ne peut citer comme représentation d'Artémis dans son sanctuaire. D'après le LIMC, l'image d'Artémis avec un polos est parfaitement courante ${ }^{59}$, et seule la répétition du motif du polos pourrait peut-être signaler une spécificité du culte thasien, du moins de l'iconographie thasienne, car en réalité ce détail vestimentaire ne nous apprend rien des fonctions d'Artémis dans son sanctuaire.

Les représentations d'Artémis sont donc plutôt rares dans le matériel votif de l'Artémision thasien et malheureusement l'iconographie, assez banale, nous apprend peu de chose sur les possibles attributions particulières de la déesse. D'autres divinités, peintes ou modelées en terre cuite, sont assez nombreuses : d'autres déesses, comme Athéna, Déméter ou Aphrodite, mais aussi des dieux, tel Dionysos, apparaissent assez souvent, sinon plus souvent qu'Artémis ellemême. En se fondant sur les seules représentations divines dans le matériel du sanctuaire, on ne l'aurait probablement pas aussitôt attribué à Artémis.

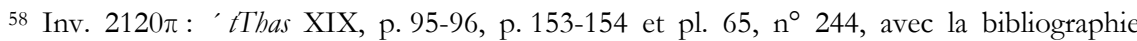
antérieure à laquelle il faut ajouter Guide de Thasos (2000), p. 289, fig. 248. Contrairement à ce que dit la notice du LIMC II, s.v. «Artemis », $\mathrm{n}^{\circ} 1364$, il ne s'agit pas de fragments de plat.

${ }^{59}$ L. KAHIL, s.v. «Artemis », LIMC II (1984), p. 738-745. 


\subsection{Artémis « Pôlô »}

Deux bases de statues du II siècle av. J.-C. portent une dédicace à Artémis « Pôlô » ${ }^{60}$. Cette épiclèse est un des rares éléments qui permettent de considérer avec quelque certitude les fonctions d'Artémis à Thasos. N. Weill insistait déjà sur le fait que la signification première du mot pôlos implique la notion de « jeunesse ». On peut donc dire qu'à Thasos, comme dans sa métropole Paros ${ }^{61}$, Artémis est la protectrice attitrée des jeunes gens, normalement sans distinction de sexes, jeunes garçons comme jeunes filles. Certaines offrandes peuvent être interprétées à la lumière de cette épiclèse.

Quelques figurines de jeunes enfants, peu nombreuses, sans oublier les osselets, en os ou en ambre, évoquent évidemment cet aspect. Certains animaux, comme les oiseaux ou les coqs, peuvent aussi être raisonnablement mis en relation avec le rôle d'Artémis protectrice de la petite enfance et initiatrice des jeunes gens ${ }^{62}$. L'interprétation des autres offrandes est problématique et encore âprement discutée. Nous suivrions volontiers, pour les figurines en terre cuite, l'interprétation que défend l'équipe de coroplathie du laboratoire de Lille, qui fait des figurines ne portant pas d'attributs divins spécifiés des représentations de mortels $^{63}$. Si l'on suit cette proposition, l'énorme masse des korés debout, très nombreuses, révélerait que la protection de la jeunesse de la cité, en particulier des jeunes filles, serait une des principales fonctions d'Artémis à Thasos. Il y aurait alors un net déséquilibre entre les offrandes de jeunes filles et de jeunes hommes, sauf si l'on considère les coupes, elles aussi nettement majoritaires dans le matériel, comme des offrandes plus spécifiquement de jeunes hommes. Cette enquête relative aux dédicaces de coupes est actuellement en cours, et nous ne proposons ici qu'une interprétation possible, mais non exclusive.

Puisque les vases à boire appartiennent à la sphère du banquet, on a tendance à les lier au monde masculin; or le groupe des vases à boire, en particulier des coupes, est tout à fait exceptionnel en proportion de l'ensemble des offrandes. Ces vases à boire étaient-ils utilisés pour des banquets, des festivités ? Force est de constater, cependant, qu'il y a très peu de cratères et d'amphores, tout aussi

${ }^{60}$ IG XII Suppl., 282-283; voir aussi n. 3.

61 Une inscription parienne de l'époque romaine impériale (IG XII, 5, Suppl. 202) atteste dans l'île un culte d'Artémis Pôlô; mais on peut se demander si ce culte n'est pas un apport de Thasos à Paros plutôt que le contraire : voir à ce sujet 't Thas III (cf. n. 3), p. 338. Sur les cultes de Paros et de Thasos, voir notamment D. BERRANGER, Recherches sur l'histoire et la prosopographie de Paros à l'époque archaïque, Clermont-Ferrand, 1992, p. 184-203.

62 St. Huysecom-Haxhi, qui vient de publier les figurines de l'Archaïsme mûr et récent (cf. n. 8), offre une synthèse intéressante, à laquelle nous devons beaucoup, sur les possibles significations des catégories de figurines offertes à Artémis : ‘tThas XXI (2009), p. 569-604. Pour les aspects évoqués ici, voir p. 572 (enfants) et p. 601-604 (animaux).

63 Laboratoire Halma-Ipel - UMR 8164. Sur leur prise de position, St. HuYsECOM, A. MuLLER, l.c. (n. 42), p. 231-247, A. Muller, l.c. (n. 34), p. 81-95, et 't thas XXI (2009), p. 583-587. 
nécessaires aux banquets. Ces grands vases auraient-ils existé mais disparu parce qu'ils étaient en bronze?

Les coupes sont aussi des objets d'usage votif. Celle d'Épictétos (Fig. 3), si la restitution de la fin de l'inscription est exacte, avait été dédiée par un homme, un certain Apollodôros, comme cela est fréquent dans les autres sanctuaires grecs ${ }^{64}$. Dans la mesure où les femmes de citoyens ne participaient pas aux banquets, dont la coupe est l'élément le plus caractéristique - il suffit de regarder pour s'en convaincre les nombreuses scènes de banquets dans l'iconographie grecque -, on peut émettre l'hypothèse que ces coupes étaient plus volontiers dédiées par des hommes. Il est vrai que le fragment de Thasos possiblement porteur d'une dédicace féminine sur une coupe de Siana - qui vient s'ajouter à cinq autres cas recensés de dédicaces de coupes faites par des femmes ${ }^{65}$ - reste un obstacle à notre démonstration tant que notre enquête n'est pas achevée.

Si l'on suppose que la plupart des coupes et des skyphoi ont été offerts à Artémis par des hommes, cela montrerait que la déesse était honorée à Thasos, comme à Brauron ${ }^{66}$, en tant que protectrice des jeunes gens des deux sexes. Le tout jeune citoyen remercierait la déesse d'avoir franchi le passage de l'éphébie à l'âge adulte, et donc son entrée dans le corps civique, en lui dédiant une coupe, car en intégrant celui-ci, le citoyen acquiert aussi le "droit au banquet ${ }^{67}$. La coupe serait ainsi l'équivalent des offrandes évoquant le monde militaire, auquel accède aussi le jeune homme quand il a achevé son initiation éphébique et qui constitue l'autre aspect important de la vie du citoyen. Artémis aurait donc à Thasos un rôle civique, que la localisation du sanctuaire, intra-muros, à proximité de l'Agora, sur l'axe principal de circulation de la cité, ne ferait que confirmer ${ }^{68}$. Mais une telle hypothèse, qui réévalue la part des offrandes masculines dans le matériel votif des sanctuaires d'Artémis, ne saurait faire oublier que la part des offrandes féminines est assurément plus importante.

\subsection{Artémis, protectrice du mariage}

Artémis veille aussi sur ce passage décisif de la vie des femmes grecques qu'est le mariage. Un certain nombre d'offrandes peut être mis en rapport avec cette étape de leur vie. La décoration de quelques vases recourt à des thèmes

64 M.L. LazZarini, Le Formule delle dediche votive nella Grecia arcaica, Rome, 1976, recense 33 dédicaces masculines sur des coupes, auxquelles s'ajoutent 14 dédicaces sur d'autres formes de vases à boire.

65 LAZZARINI, o.c. (n. 64), cat. 12, 151, 152, 387, 648.

66 Voir notamment LUNDGREEN, l.c. (n. 36).

${ }^{67}$ L'expression est de St. HuyseCOM-HAXHI, 't thas XXI (2009), p. 604, qui interprète ainsi les figurines de banqueteurs.

68 Sur la localisation des Artémisia en rapport avec les fonctions d'Artémis, voir S. GUETTEL COLE, Landscapes, Gender and Ritual Space. The Ancient Greek Experience, Berkeley, 2004, p. 178197. 
qui semblent faire allusion à cet important moment : on citera comme exemples une scène possible de cérémonie de mariage sur une coupe de Siana attribuée au Peintre $C^{69}$ (Fig. 23) et une procession de femmes sur une grande lékanè attique à figures noires assignable au même peintre ${ }^{70}$. Les vases à parfum peuvent aussi être envisagés comme évoquant le cérémonial du mariage, dans lequel le parfum jouait un rôle important.

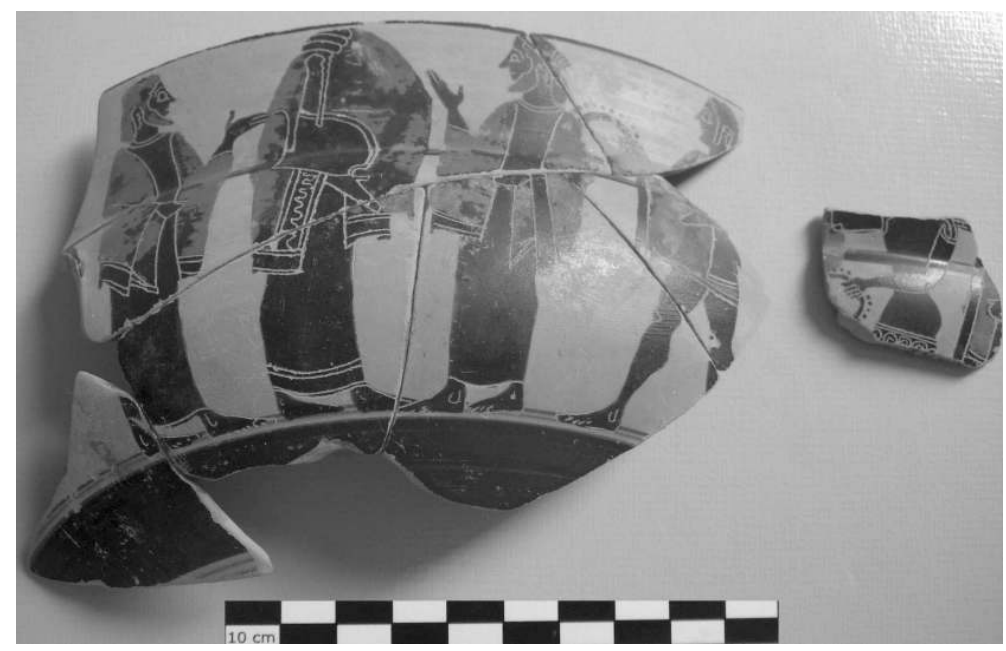

Fig. 23

Certains bijoux présents à l'Artémision thasien sont, d'une manière évidente, une évocation de la femme dans ses atours les plus précieux, mais seul le diadème est susceptible de faire directement référence à la cérémonie du mariage. Les autres éléments de parure ne font que témoigner d'une fréquentation féminine du sanctuaire.

Artémis protège ensuite la femme dans son statut social d'épouse. Les offrandes d'objets liés au travail de la laine, épinétra et pesons, peuvent être envisagés sous deux aspects. Le tissage est l'activité essentielle qu'apprend la jeune fille durant son initiation, et certains voient dans de telles offrandes une allusion à la domestication de la jeune fille devenue femme ${ }^{71}$; ce serait donc la fonction de protectrice des jeunes filles qui serait à privilégier. Mais on peut aussi voir dans ces objets qui évoquent la femme dans son travail quotidien une célébration de la

${ }^{69}$ Inv. $2440 \pi$ : BCH 82 (1958), p. 812, fig. 13; H. BrIjDER 1983 (n. 11), p. 239-240, n 45, pl. $13 \mathrm{~d}$.

${ }^{70}$ Inv. $2074 \pi$ : BCH 82 (1958), p. 813 fig. 14; J.D. BEAZLEY, Paralipomena 25, 212 (erreur pour 112) bis.

71 Ainsi Y. MORIzoT, « Offrandes à Artémis pour une naissance, autour du relief d'Achinos », in V. DASEn (éd.), Naissance et petite enfance dans l'Antiquité (Actes du colloque de Fribourg 28/111/12/2001), Fribourg, 2004, p. 167-168; S. GuETTEL COLE, o.c. (n. 68), p. 214. 
déesse de la part d'une épouse désirant la remercier du succès de son activité principale au sein de l'oikos. D'après l'équipe de recherche de Lille 3 , les figurines de dames assises pourraient être autant d'évocations de la femme dans son statut d'épouse $^{72}$.

\subsection{Artémis « Eileithyia »}

Il s'agit là de l'autre épiclèse d'Artémis connue à Thasos ${ }^{73}$. Eileithyia, divinité qui veille sur les accouchements, peut être honorée seule, mais elle est souvent associée à Artémis, au point que son nom est devenu une épiclèse assez courante de la déesse $\mathrm{e}^{74}$. C'est sans doute Artémis comme protectrice des accouchées et des jeunes mères qui est honorée par les quelques figurines du type courotrophe. Les statuettes qui représentent des nains ou d'autres créatures apotropaïques sphinges, sirènes - ont pu être aussi dédiées pour demander l'aide de la déesse au moment de l'accouchement, pour la mère et pour son enfant ${ }^{75}$.

Nous savons que, dans les grands Artémisia de Brauron et de Milet, pour lesquels nous disposons des inventaires, les jeunes femmes dédiaient fréquemment leurs vêtements en remerciement d'un accouchement heureux. La ceinture est particulièrement symbolique de ce moment difficile. Le décret en l'honneur d'Épié témoigne de cette pratique à l'Artémision de Thasos. De ces vêtements disparus, fibules et éléments de ceintures sont peut-être les témoins. Les coffres, qui pouvaient constituer des offrandes à part entière en tant que biens importants de la femme, comme le montrent sur les vases les images de gynécée, étaient peut-être aussi à Thasos, comme à Brauron, remplis de vêtements.

\section{Conclusion}

Les sanctuaires d'Artémis présentent souvent un matériel votif témoignant d'une vénération féminine à la déesse qui accompagne les étapes importantes de la vie de la femme grecque, un des meilleurs exemples étant fourni par le sanctuaire de Brauron ${ }^{76}$. L'Artémision de Thasos ne fait pas exception. Les figurines de terre cuite peuvent être sans doute, plus que les vases, plus clairement

\footnotetext{
72 St. Huysecom, A. Muller, l.c. (n. 42), p. 240-242; ’ tThas XXI (2009), p. 583-587.

73 Pour le décret en l'honneur d'une certaine Épié, qui atteste cette épiclèse, voir Fr. SALVIAT, BCH 83 (1959), p. 362-392.

${ }^{74}$ S. Pingiatoglou, Eileithyia, Würzburg, 1981, p. 98-119; les p. 118 et 119 sont consacrées au culte de Thasos.

75't thas XXI (2009), p. 600-601.

${ }^{76}$ Deux études récentes sur les rituels pratiqués et les offrandes faites dans ce sanctuaire: E. VIKELA, "The Worship of Artemis in Attica: cult, places, rites, iconography ", in N. KALTSAS, A. SHAPIRO (éds), Worshipping Women. Ritual and Reality in Classical Athens, Athènes, 2008, p. 79-87; I. NiELSEN, "The Sanctuary of Artemis Brauronia. Can architecture and iconography help to locate the settings of the rituals? ", in FisHer-HANSEN, POULSEN (éds), o.c. (n. 36), p. 83-126.
} 
rattachées à une vénération féminine. Mais des séries d'objets très richement fournies, comme celle des coupes et des skyphoi, renvoient plutôt à la sphère masculine. Une inscription témoigne aussi clairement d'une fréquentation masculine du sanctuaire ${ }^{77}$, qui n'était probablement pas si négligeable qu'on le pense parfois.

Les grandes statues de terre cuite sont des offrandes rares et d'une grande qualité : peut-être s'agissait-il d'offrandes familiales en l'honneur de la déesse, comme l'étaient, par exemple, les groupes de statues votives de Généléos et de Chéramyès à l'Héraion de Samos. D'autres sanctuaires ont livré des fragments de statues de terre cuite grandeur nature ou presque, et on a parfois déjà proposé de les interpréter comme des offrandes faites par des parents pour évoquer d'une façon idéale leurs enfants et remercier les dieux d'avoir accordé à ceux-ci leur protection ${ }^{78}$. Ce sont donc les jeunes gens, des deux sexes, et leurs parents, ainsi que les femmes mariées et les jeunes mères, qui semblent avoir fréquenté le plus assidûment le sanctuaire de Thasos.

Nous avons tenté de donner un sens à un certain nombre d'offrandes, mais la grande masse du répertoire, assez banal, évoque moins Artémis qu'une divinité féminine en général. L'extraordinaire variété et la grande richesse du matériel votif de l'Artémision, par sa seule présence, ne permettrait que difficilement l'identification du sanctuaire, si nous n'avions pas des témoignages écrits explicites. Les offrandes qui peuvent être rattachées à plusieurs aspects de la personnalité d'Artémis posent des problèmes d'interprétation : aucune fonction précise de la déesse ne peut être mise en avant d'une manière évidente par l'étude de ce matériel. Ces problèmes d'interprétation, nous les devons à la grande souplesse des pratiques religieuses grecques et à la malléabilité de la signification des objets votifs en fonction du projet du dédicant. Ce n'est pas, à notre avis, que ces offrandes n'aient pas été porteuses de sens, mais ce sens est souvent perdu pour nous aujourd'hui, si rien d'objectivement clair ne l'explicite.

Anne TICHIT

Laboratoire Halma-Ipel - UMR 8164

École française d'Athènes

Didotou, 6

GR - 10680 ATHÈNES

anne.tichit@efa.gr
Jean-Jacques MAFFRE

Institut d'Études grecques Université de Paris IV - Sorbonne 1 , rue V. Cousin

FR - 75230 PARIS Cedex 05 Jean-Jacques.Maffre@paris-sorbonne.fr

\footnotetext{
77 BCH 86 (1962), p. 592, n 13 : il s'agit d'une dédicace d'un certain Philôn, fils de Phanagorès.

78 Voir par exemple le cas du sanctuaire de Déméter et Korè à Corinthe : N. BoOKIDIS, «Large-Scales Terracottas and the Cult of Demeter and Kore in Corinth », Kernos, Suppl. 23 (n. 1), p. 225-237. L'auteur cite, sans toutefois s'y rallier, l'opinion d'A. Chaniotis, qui estime que ces statues «pouvaient avoir été dédiées par des parents en remerciement pour le bien-être de leurs enfants » (p. 236).
} 


\section{Liste des figures}

Fig. 1. Plan du secteur Nord-Est de l'Agora de Thasos: au Sud, l'Artémision et ses deux terrasses (nos 52-55). - Plan ÉfA, M. Wurch-Koželj.

Fig. 2: Fragment inédit de coupe attique à vernis noir portant la dédicace APTE[MI $\Delta \mathrm{I}] .1^{\text {re }}$ moitié du ve s. av. J.-C. - (C) G. Naessens (Halma-Ipel-UMR 8164).

Fig. 3. Coupe attique à figures rouges, attribuée à Épictétos (avec la possible dédicace d'Apollodôros). - Cliché ÉfA, Ph. Collet.

Fig. 4. Fragments d'une coupe de Siana (inv. 75.A.489 $\alpha$ ) portant une inscription. (C) G. Naessens (Halma-Ipel-UMR 8164).

Fig. 5. Fragment d'une coupe attique à vernis noir portant l'inscription IPH. -CC G. Naessens (Halma-Ipel-UMR 8164).

Fig. 6. Pesons en terre cuite de formes variées. - Cliché J.-J. Maffre.

Fig. 7.. Fragment d'un épinétron attique à figures noires (inv. 59.3403), fin du VIe s. av. J.-C. : scène d'intérieur. - Cliché J.-J. Maffre.

Fig. 8. Fragments d'un épinétron attique à figures noires (inv. 59.3392 / 59.3396 / 59.3404 / 59.3406 / 59.3411 / 59.3412) : scène de course de chars. - Cliché J.-J. Maffre.

Fig. 9. Diadème en or décoré. - Cliché ÉfA, Ph. Collet; dessin ÉfA.

Fig. 10. Disques et fibules en ivoire travaillé (long. de la fibule bilobée : env. $8 \mathrm{~cm}$; diam. des disques : $c a .8 \mathrm{~cm}$ pour les grands, $4 \mathrm{~cm}$ pour le petit). - Cliché ÉfA, Ph. Collet.

Fig. 11. Support de miroir sculpté en bronze (inv. 1464). H. 19 cm. - Cliché ÉfA, Ph. Collet.

Fig. 12. Fragments du torse d'une statue féminine en terre cuite de style dédalique (inv. 6351). Cliché ÉfA, É. Sérafis.

Fig. 13. Figurines animales de terre cuite de production corinthienne: tortue (inv. 2050 $)$, bélier (inv. $3913 \pi)$, lièvre $(2383 \pi)$ et lion $(3880 \pi)$. - Clichés J.-J. Maffre.

Fig. 14. Figurine de terre cuite : la «Dame au Polos» (inv. F 15013/r1936). - Cliché ÉfA, Ph. Collet.

Fig. 15. Sélection de vases à parfum : alabastre à figures rouges (inv. $3131 \pi$ ), lécythes à figures

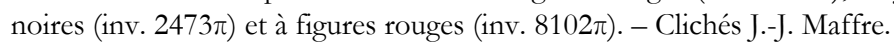

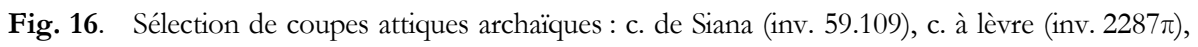

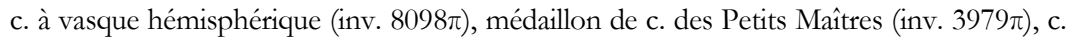
de Cassel (inv. 75.A.3686), c. à figures rouges (inv. 4861 $)$ ). - Clichés J.-J. Maffre.

Fig. 17. Plaquette de décor appliqué en ivoire. - Cliché ÉfA, Ph. Collet.

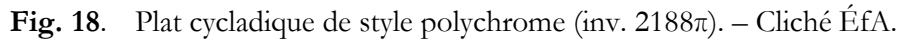

Fig. 19. Plat gréco-oriental de style polychrome (inv. 2182 $)$ ). Cliché ÉfA.

Fig. 20. Relief moulé représentant la Potnia Thérôn (?), ailes déployées (inv. F 24020/л3883). Ateliers thasiens. - Cliché ÉfA.

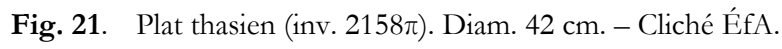

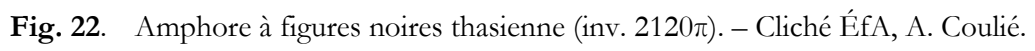

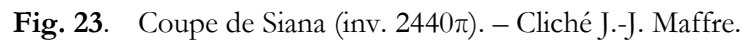

